\title{
AVALIAÇÃO DE GENÓTIPOS DE TRIGO PARA A REGIÃO DO VALE DO PARANAPANEMA NO QUADRIÊNIO 1981-84 ( $\left.{ }^{1}\right)$
}

\author{
JOĀO CARLOS FELfCIO $(2,5)$, \\ CARLOS EDUARDO DE OLIVEIRA CAMARGO $(2,5)$, \\ JOSÉ GUILHERME DE FREITAS $(2,5)$, \\ ANTONIO WILSON PENTEADO FERREIRA FILHO $\left({ }^{2}\right)$, \\ BENEDITO DE CAMARGO BARROS $(3,5)$ \\ e MARCELO BENTO PAES DE CAMARGO $(4,5)$
}

\begin{abstract}
RESUMO
Estudou-se, durante o quadriênio 1981-84, o comportamento de novos cultivares de trigo para solos com e sem alumínio na camada arável, conjuntamente com cultivares comerciais, avaliando-se: produtividade de grãos, reaçōes aos agentes de ferrugem do colmo, da folha e Helminthosporium sp., bem como as condiçôes climáticas da região sudoeste paulista. As condiçōes climáticas foram muito diversificadas, não se podendo fazer comparação dos resultados de um ano com outro, em razão de serem usados diferentes cultivares. Os considerados suscetíveis à toxicidade de alumínio não apresentaram destaque para a produtividade de grão em comparação à testemunha, BH 1146; entretanto, entre os tolerantes, destacaram-se PAT 72247, CEP 7780 e BH 1146. Com relação à resistência à ferrugem do col-
\end{abstract}

(1) Com verba suplementar do acordo do trigo entre as Cooperativas de Produtores Rurais do Vale do Paranapanema e a Secretaria de Agricultura e Abastecimento, por intermédio do Instituto Agronômico. Recebido para publicação em 18 de fevereiro de 1986.

(2) Seção de Arroz e Cereais de Inverno, Instituto Agronômico (IAC), Caixa Postal 28, 13001 Campinas (SP).

(3) Seção de Doenças das Plantas Alimentícias Básicas e Olerícolas, do Instituto Biológico, Caixa Postal 70, 13001 Campinas (SP).

(4) Seçäo de Climatologia Agrícola, Instituto Agronômico, IAC.

(5) Com bolsa de suplementação do CNPq. 
mo, sobressaíram: Anahuac, Sparrow"S", CEP 74138, CEP 7780, R 30464-77, CNT 8, El Pato e INIA 66. Para a ferrugem-da-folha, destacou-se com boa resistência, o 'IAPAR 1 Mitacoré. Já o 'Alondra 46', que apresentava boa resistência a essa ferrugem no início do período, revelou-se altamente suscetível a partir de 1983. Para a helmintosporiose, houve variação na sua ocorrência, em razão de estar correlacionada ao período de exposição da planta à umidade de pós-inoculação do patógeno.

Termos de indexação: trigo, genбtipos, produtividade de gräo, ferrugem do colmo e da folha, helmintosporiose.

\section{INTRODUÇÃO}

A distribuição regional das culturas e a variabilidade dos rendimentos anuais são dependentes, em grande parte, do clima. Estudos visando determinar as relaçōes entre adaptação, rendimento, zonearrento e clima têm merecido especial atenção para o equacionamento e solução das adversidades climráticas que a cultura do trigo encontra no Brasil (MOTA, 1982). As áreas utilizadas para esse cultivo são de regiōes com grande variação anual de temperatura e de relativamente escassas precipitações pluviais (KALCKMANN et alii, 1965).

No rendimento de uma cultura, influem todas as condições ambientes que afetam o crescimento da planta. A capacidade do rendimento pode estar expressa por características morfológicas de cada planta, cujo desenvolvimento deve ocorrer em um ambiente favorável e não limitado por fatores adversos, como frio, umidade, seca, pragas e doenças (POEHLMAN, 1974).

Atualmente, no Estado de São Paulo, a maior área de cultivo de trigo se encontra no Vale do Paranapanema, favorável ao desenvolvimento da cultura, embora prejudicada, em alguns anos, pela seca no outono (EMBRAPA, 1984).

$O$ deslocamento da tradicional área paulista de trigo, a região sul, para - Vale do Paranapanema, sudoeste, ecologicamente melhor para a cultura (DUARTE, 1974), deveu-se principalmente às condiçōes dos solos, de baixa para alta fertilidade.

A indicação de cultivares mais adaptados a solo de maior ou menor fertilidade, bem como com tolerância ao $\mathrm{Al}^{3+}$, pode ser urra medida benéfica, tendo em vista o custo dos insurros. Cultivares de trigo como Maracaí (IAC-17) e Xavantes (IAC-18) apresentam adaptação às regiōes triticolas paulistas, boa produtividade de grãos, resistência às principais doenças (FELíclO et alii, 1983) e tolerância ao $\mathrm{Al}^{3+}$.

As ferrugens do colmo e da folha chegam a ser fatores limitantes na produção de trigo, exigindo, muitas vezes, o controle químico, prática geralmente onerosa e nem sempre satisfatória. Grande diversidade na população patogênica, não só pelas novas formas de virulência corro pela mudança de prevalência, nos últimos levantamentos, realizados por COELHO (1984) e BARCELOS \& AITA 
(1984), faz com que a seleção de variedades com resistência às raças dos patógenos prevalentes seja uma preocupação constante da pesquisa, visando, com isso, minimizar os riscos decorrentes dessas importantes doenças.

Com relação às manchas foliares, causadas principalmente por Helminthosporium sp., foram estimadas perdas de cerca de 20 e $50 \%$, respectivamente, em anos considerados normais e chuvosos, no Sul de São Paulo, com uma variedade suscetivel (BARROS et alii, 1982). Experimentos conduzidos nos últimos anos em Palotina (PR), com cultivares de trigo de origem mexicana, demonstraram perdas causadas por helmintosporiose de até $36 \%$ da produção, valor que representou $86 \%$ das perdas totais, causadas por somente um dos componentes do complexo de doenças (OLIVEIRA \& GOMES, 1984).

Este trabalho foi conduzido de 1981 a 1984, com o objetivo de avaliar o desempenho quanto à produtividade de grãos de diversos cultivares de trigo na região do Vale do Paranapanema, SP, em função da tolerância ou suscetibilidade ao $\mathrm{Al}^{3}+$ e da ocorrência de doenças.

\section{MATERIAL E MÉTODOS}

Diversos cultivares de trigo foram ensaiados em três grupos distribuídos quanto à tolerância ou suscetibilidade à acidez do solo, de acordo com a metodologia utilizada por MUZZILLI et alii (1978) e/ou CAMARGO \& OLIVEIRA (1981) na camada arável, reunindo o terceiro grupo os cultivares em cultivo comercial.

Os cultivares que compuseram cada um dos grupos foram os seguintes:

GRUPO 1. Cultivares suscetiveis ao $\mathrm{Al}^{3+}$

Anahuac, Cocoraque, Candeias, IAPAR 3 (Aracatu), IAC 15, Klein Toledo, Diamante INTA, IAPAR 6 (Tapejara), Jandaia, OC 731070, Pavão, OC 804, OC 807, OC 808, Sparrow"S", MS 80-1, Ld 7821, IA 78103, IAC 23 (Tocantins), INDUSEM 3, OCEPAR 7, BR 11, OCEPAR 6, OC 8120, Alondra 46, OC 816, OCEPAR 12, MS 7878, GD 798, GD 799, PF 79606, IAC 161, Ld 7815, OC 8112, R 30147-77, IAC 162, Glennson M 81, IA 7998, PF 781148 e PF 79475, sendo considerados testemunhas para a tolerância à acidez do solo os cultivares: $\mathrm{BH}$ 1146 e IAC 24 (Tucuruî).

GRUPO 2. Cultivares tolerantes ao $\mathrm{Al}^{3}+$

Alondra 46, BH 1146, CEP 74138, CEP 74162 (Sulino), CEP 7672, CEP 7780, IAC 5 (Maringá), IAC 21 (Iguaçu), IAC 22 (Araguaia), IAC 24 (Tucuruí), IA 791, Macuco, OC 814, OC 8110, OC 8123, PAT 72247, PF 75171, PF 75388, PF 75418, PF 7815, BR 13 (PF 782027), R 30464-77, R 30469-77, OC 8148, OC 8112, IAC 160, IAC 72, PG 8115, PG 8116, B 7944, OC 8122 e CEP 7951. 


\section{GRUPO 3. Cultivares comerciais}

Alondra 46, BH 1146, CNT 7, CNT 8, CNT 9, Confiança, El Pato, IAC 5 (Maringá), IAC 13 (Lorena), IAC 17 (Maracaí), IAC 18 (Xavantes), IAPAR 1 (Mitacoré), INIA 66, Itapua 5, Jupateco 73, LA 1549, Moncho BSB, Nambu, Paraguay 281, PAT 24, PAT 7219, BR 1, BR 2, Tucano, Anahuac, Charrua, Cocoraque, IAPAR 3 (Aracatu), Jandaia, PAT 7392, IAC 21 (Iguaçu), IAC 23 (Tocantins) e IAC 24 (Tucuruí).

Os ensaios foram instalados no quadriênio de 1981 e 1984, sempre na segunda quinzena de abril, nas localidades de Maracaí, Palmital, Pedrinhas Paulista e São José das Laranjeiras, com predominância de solos de boa fertilidade, mesclados com solos de baixa fertilidade que apresentavam problemas de alumínio tóxico.

Retiraram-se amostras do solo dos locais em estudo e, de acordo com os resultados analíticos, efetuou-se a adubação seguinte: sulfato de amônio com $20 \%$ ce $\mathrm{N}$, superfosfato simples corr $20 \%$ de $\mathrm{P}_{2} \mathrm{O}_{5}$ e cloreto de potássio com $60 \%$ de $\mathrm{K}_{2} \mathrm{O}$ aplicados a lanço antes da semeadura e, posteriormente, incorporados ao solo. As quantidades de fertilizantes aplicadas nos diferentes locais basearam-se nas tabelas de adubação e calagem do Instituto Agronômico (CAMPINAS, 1977).

O delineamento empregado foi de blocos ao acaso com quatro repetições por local, utilizando-se a análise conjunta dos experimentos em blocos ao acaso com tratamento comum (GON.ES, 1963).

As parcelas de cada ensaio foram constituídas de cinco linhas de $5 \mathrm{~m}$ de comprimento, distanciadas de $0,20 \mathrm{~m}$, deixando-se um espaçamento lateral, entre cada parcela, de $0,60 \mathrm{~m}$, e de $1 \mathrm{~m}$ entre os blocos. A semeadura foi efetuada na base de 80 sementes por metro linear de sulco, equivalendo a 400 sementes por metro quadrado.

Os dados termopluviométricos diários relativos aos periodos considerados foram obtidos do Posto Meteorológico da Fazenda Canadá (lat. $22^{\circ} 40^{\prime} \mathrm{S}$.; long. $50^{\circ} 25^{\prime} \mathrm{W}$; alt. $569 \mathrm{~m}$ ) no município de Assis, SP, mantido em colaboração corr o IAC, podendo ser considerado como representativo de toda a região estudada.

A caracterização da disponibilidade hídrica no solo foi feita através de balanços hídricos decendiais segundo o método de THORNTHWAITE \& MATHER (1955), considerando $125 \mathrm{~mm}$ como capacidade de retenção de água no solo. Calcularam-se os balanços hídricos para os meses de março a setembro, em 1981-1984.

Para avaliar o comportamento dos cultivares com relação às principais doenças em condições naturais de infecção, foram empregadas escalas de leitura. Para a ferrugem do colmo (Puccinia graminis Pers. forma sp. tritici Eriks, et Henn.) e ferrugem da folha (Puccinia recondita Rob. ex. Desm. forma sp. tritici 
Eriks), foram feitas avaliações em planta adulta usando a escala modificada de Cobb, empregada por SCHRAM et alii (1974), e que é composta por um número, estimando o ataque da moléstia no colmo e na folha, acrescido de uma letra simbolizando o tipo de reação: $S$ = suscetível (uredossoro grande, coalescente, sem necrose e sem clorose); $M S=$ moderadamente suscetível (uredossoro médio); $\mathrm{M}=$ intermediário (diversos tipos de reação); $\mathrm{MR}=$ moderadamente resistente (uredossoro pequeno); $\mathrm{R}$ = resistente (uredossoro minúsculo rodeado de áreas necróticas).

Em outras doenças, como o complexo septoriose mais helmintosporiose (Septoria sp. e Helminthosporium sp.), ocorrentes nas folhas, as infecções foram estimadas em porcentagem de área foliar infectada de 0-100, observando-se as folhas superiores das plantas.

Por ocasião da colheita, tomaram-se as cinco linhas de cada parcela, com área útil de $5 \mathrm{~m}^{2}$. Submeteram-se os dados de produção à análise conjunta de experimentos em blocos ao acaso com tratamentos comuns (GOMES, 1970), subdivididos em grupos de cultivares, quanto à sua tolerância e suscetibilidade ao $\mathrm{Al}^{3}+e$ os cultivares comerciais, acrescentando-se os cultivares-padrōes mais importantes.

\section{RESULTADOS E DISCUSSÃO}

A germinação nas diferentes parcelas dos experimentos foi muito boa, assegurando bom estande inicial. A ocorrência de doenças, como a ferrugem do colmo, a ferrugem da folha e a helmintosporiose, mostrou grande variação na intensidade de ataque, sobretudo com relação à última, provavelmente devido aos períodos mais prolongados de chuva verificados no período, nos meses de maio a julho. Foram quatro anos climaticamente diferentes, com precipitações irregulares (Figura 1), apresentando os anos de 1982 e 1983 maiores intensidades de chuvas em maio, junho e julho do que normalmente ocorre na regiăo Oeste paulista, limitrofe norte do Estado do Paraná. O aumento da severidade da doença em 1982 e 1983 estaria correlacionada com a duração do período de umidificação pós-inoculação (UPI), de acordo com LUZ (1982).

\section{Ensaio de cultivares de trigo para solos sem acidez}

No quadro 1, encontram-se os dados de produção de grãos de trigo dos cultivares com suscetibilidade ao alumínio, fazendo exceģão os cultivares $\mathrm{BH} 1146$ e IAC 24, que são tolerantes. Através do método de análise conjunta de experimentos em blocos ao acaso com tratamentos comuns (GOMES, 1970), verificou-se um efeito altamente significativo para tratamento $(F 42,36)$ quando se consideraram as médias do quadriênio. $\mathrm{Na}$ análise conjunta dos experimentos plantados em diferentes localidades em cada ano, verificaram-se, no período, efeitos significativos para tratamentos, anos e interação tratamentos $x$ anos, demonstrando que em cada ano estudado os cultivares se apresentaram com um comportamento diferenciado entre si, possivelmente devido a variações climatológicas. 
A maior média dos experimentos nos quatro anos estudados foi de $1.597 \mathrm{~kg} / \mathrm{ha}$, em 1981, enquanto a menor foi de $1.054 \mathrm{~kg} / \mathrm{ha}$, em 1983 . Essas variações se devem, principalmente, às condições climatológicas altamente favoráveis em 1981: boa precipitação durante a segunda quinzena de abril e início de maio, pequena estiagem até o final do mês, quando se reiniciaram novas precipitações até meados de junho, encerrando-se o ciclo da cultura em agosto, com estiagem. O contrário ocorreu em 1982 e 1983: em maio e junho, houve um aumento significativo das precipitações, com períodos prolongados de chuva, acarretando grande incidência de moléstias, como as do complexo de manchas foliares, afetando principalmente as folhas e as espigas. Já em 1984, com estiagens ainda mais prolongadas, as doenças não se manifestaram (Figura 1), impossibilitando a leitura em condiçōes de campo.
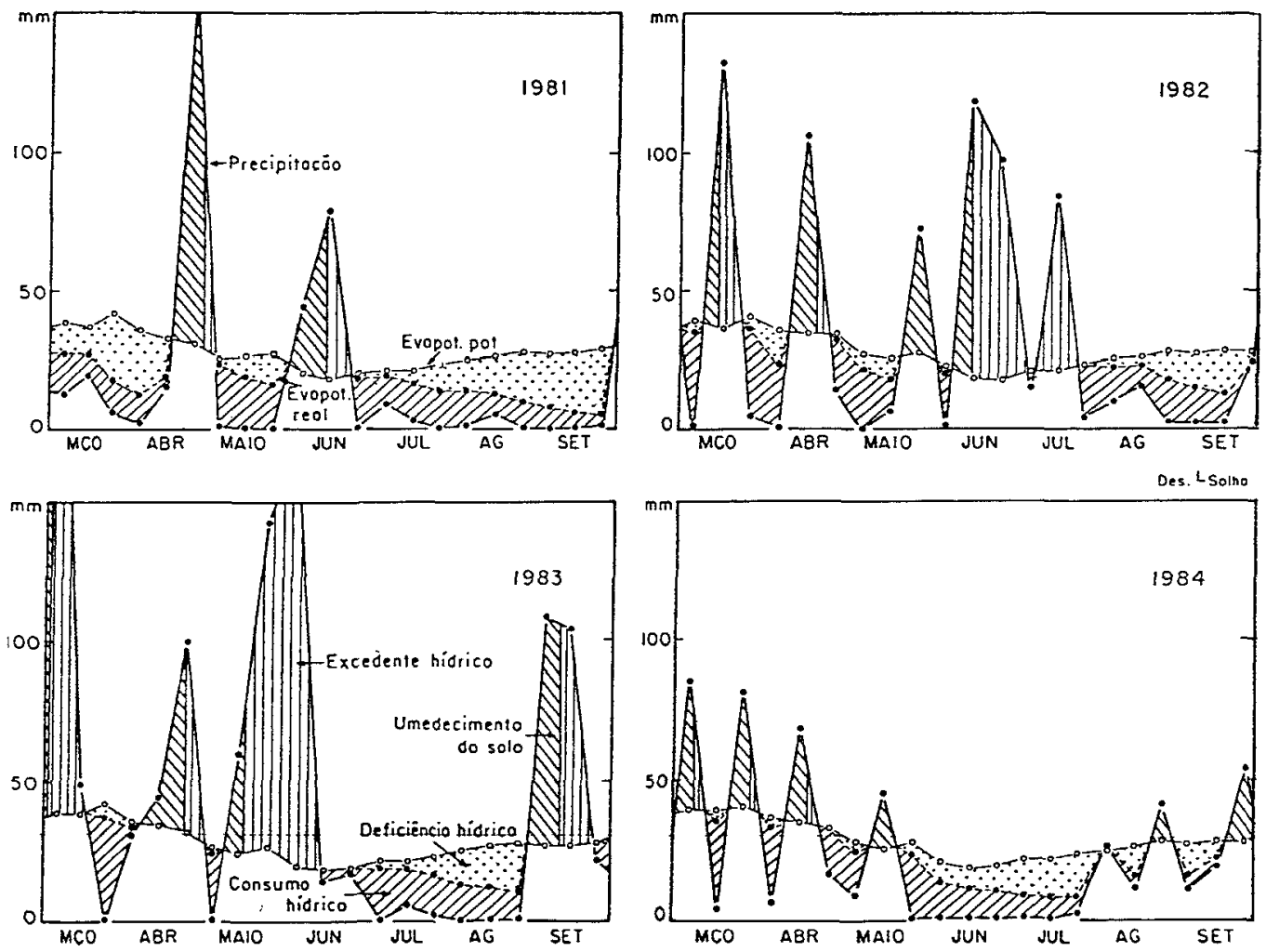

FIGURA 1. Balanço hídrico decendial pelo método de THORNTHWAITE \& MATHER (1955) $(125 \mathrm{~mm})$, referente ao período de 1981 a 1984 para a região de Assis, SP. 


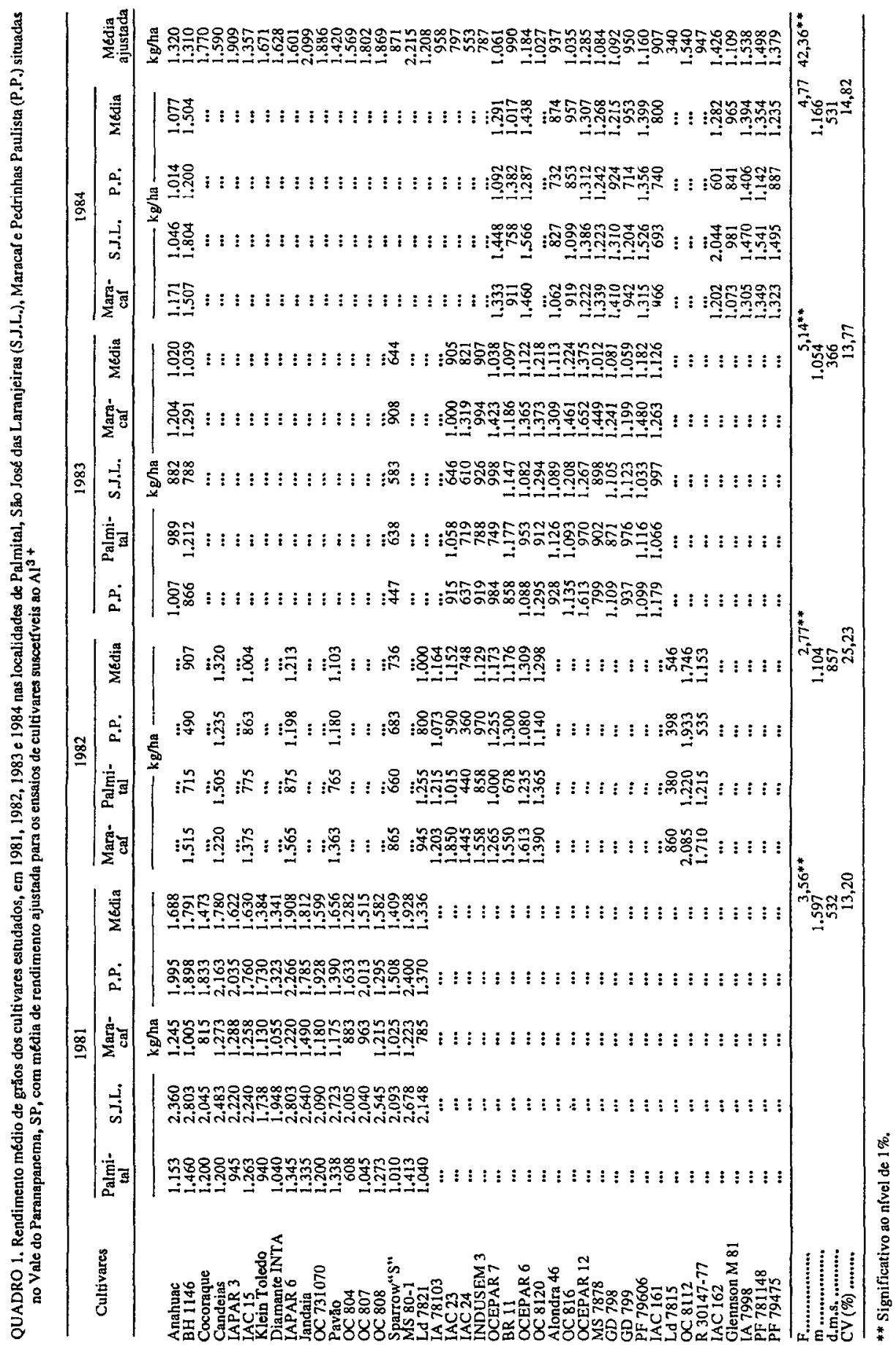


Ao analisarmos a reação dos cultivares com relação à ferrugem do colmo - Quadro 2 - verifica-se que o BH 1146 apresentou suscetibilidade em todo o período, destacando o 'Anahuac' e o 'Sparrow"S" com boa resistência à doença, em razão de terem apresentado essa estabilidade em todo o período. Com relação à ferrugem da folha - Quadro 3 - a grande maioria dos cultivares apresentou suscetibilidade, sem, contudo, causar danos maiores a sua produtividade de grãos. Entretanto, no estudo da reação às manchas foliares, causadas principalmente por Helminthosporium sativum - Quadro 4 - todos se apresentaram suscetíveis, em maior ou menor grau de infecção, estando a intensidade da doença interligada às condições climáticas dos períodos estudados.

QUADRO 2. Dados mCtlios (Escala de Cobb modificada) das reaçסes a ferrugem do colmo (Puccinia graminis tritict) obtidos pelos cultivares avaliados em 1981, 1982 e 1983 nas localidades de Maracaf, Sũo Jose das Laranjcirus, Pedrinhats l'aulista e Pal mital, nos crsujos de cultivares suscetiveis ao $\mathrm{Al}^{3+}$

\begin{tabular}{|c|c|c|c|c|c|c|c|c|c|}
\hline \multirow[b]{2}{*}{ Cultivares } & \multicolumn{2}{|c|}{1981} & \multicolumn{3}{|c|}{1982} & \multicolumn{4}{|c|}{1983} \\
\hline & $\begin{array}{l}\text { São Jose das } \\
\text { Laranjeiras }\end{array}$ & Maracal & Maracaf & $\begin{array}{l}\text { Pedrinhas } \\
\text { Paulista }\end{array}$ & Palmital & $\begin{array}{l}\text { São Iost das } \\
\text { Laranjeiras }\end{array}$ & Maracaf & $\begin{array}{l}\text { Pedrinhas } \\
\text { Paulista }\end{array}$ & Palmital \\
\hline Anahuac & $i$ & ' & $\ldots$ & $\cdots$ & $\ldots$ & 0 & t & 0 & 0 \\
\hline BH 1146 & $10 \mathrm{~s}$ & $20 \mathrm{~S}$ & $90 S$ & $80 S$ & 605 & $40 \mathrm{~S}$ & 205 & 10 & $10 \mathrm{~s}$ \\
\hline Cocoraque & 0 & 0 & $\cdots$ & $\cdots$ & $\cdots$ & $\cdots$ & $\cdots$ & $\cdots$ & $m$ \\
\hline Candeias & 0 & 0 & $0-10 S$ & $5 S$ & 0 & $\cdots$ & $\cdots$ & $\cdots$ & $\cdots$ \\
\hline IAPAR 3 & $\mathrm{t}$ & 0 & $\ldots$ & $\ldots$ & $\ldots$ & $\ldots$ & $\cdots$ & $\cdots$ & $\cdots$ \\
\hline IAC 15 & 0 & 0 & $20 \mathrm{MR}$ & $20 \mathrm{MR}$ & $20 \mathrm{~S}$ & $\ldots$ & $\cdots$ & $\cdots$ & $\cdots$ \\
\hline Klein Toledo & 0 & 0 & $\ldots$ & $\ldots$ & $\cdots$ & $\cdots$ & $\cdots$ & $\cdots$ & $\cdots$ \\
\hline Diamante INTA & 0 & 0 & $\cdots$ & $\ldots$ & $\cdots$ & $\ldots$ & $\cdots$ & $\cdots$ & $\cdots$ \\
\hline IAPAR 6 & 0 & 0 & 0 & $20 \mathrm{~S}$ & $5 S$ & $\cdots$ & $\cdots$ & $\cdots$ & $\cdots$ \\
\hline Jandaia & $t$ & tMS & $\cdots$ & $\cdots$ & $\cdots$ & $\cdots$ & $\cdots$ & $\cdots$ & $\cdots$ \\
\hline OC-731070 & 0 & 0 & $\cdots$ & $\ldots$ & $\cdots$ & $\cdots$ & $\cdots$ & $\cdots$ & $\ldots$ \\
\hline Pavăo & $\mathbf{t}$ & $t$ & 0 & $10 \mathrm{MR}$ & 0 & $\cdots$ & $\cdots$ & $\cdots$ & $\cdots$ \\
\hline OC 804 & 0 & 0 & $\cdots$ & $\cdots$ & $\cdots$ & ... & $\cdots$ & $\cdots$ & $\cdots$ \\
\hline OC 807 & 5 & $10 \mathrm{~S}$ & $\cdots$ & $\cdots$ & $\cdots$ & $\cdots$ & $\cdots$ & $\cdots$ & $\cdots$ \\
\hline OC 808 & t & $t$ & $\ldots$ & $\cdots$ & $\cdots$ & $\cdots$ & $\cdots$ & $\cdots$ & $\cdots$ \\
\hline Sparrow "S" & 0 & 0 & 0 & 0 & 0 & 0 & 0 & $\ldots$ & 0 \\
\hline MS 80-1 & $i$ & $t$ & $\cdots$ & $\cdots$ & $\cdots$ & $\ldots$ & $\cdots$ & $\cdots$ & $\cdots$ \\
\hline Ld 7821 & 0 & 0 & 0 & $\mathrm{SR}$ & 0 & $\cdots$ & $\cdots$ & $\cdots$ & $\cdots$ \\
\hline IA 78103 & $\ldots$ & $\ldots$ & 0 & $5 \mathrm{R}$ & 0 & $\ldots$ & $\ldots$ & $\ldots$ & $\ldots$ \\
\hline IAC 23 & ... & $\cdots$ & 10MS & $30 \mathrm{~s}$ & $10 \mathrm{~S}$ & $30 \mathrm{~s}$ & $20 S$ & $\cdots$ & $30 \mathrm{~S}$ \\
\hline IAC 24 & $\ldots$ & $\ldots$ & $20 \mathrm{MR}$ & 20MR & $10 \mathrm{~S}$ & $20 \mathrm{~S}$ & $t$ & $\cdots$ & $20 \mathrm{~S}$ \\
\hline INDUSEM 3 & ... & $\ldots$ & 0 & 0 & 0 & $30 \mathrm{~S}$ & $5 S$ & $\ldots$ & $20 S$ \\
\hline $10 C 813$ & $\ldots$ & $\ldots$ & 0 & SMR & 0 & $t$ & 0 & $\cdots$ & $10 S$ \\
\hline MS 7810 & $\ldots$ & $\ldots$ & 0 & tS & 0 & $10 \mathrm{~S}$ & $5 S$ & $\cdots$ & $40 S$ \\
\hline OCEPAR 6 & $\cdots$ & $\cdots$ & $\cdots$ & $\cdots$ & $\cdots$ & $10 \mathrm{~s}$ & $\mathrm{t}$ & $\cdots$ & $30 \mathrm{MS}$ \\
\hline OC 8120 & $\ldots$ & $\ldots$ & tS & $30 \mathrm{~S}$ & $t$ & $30 s$ & 5s & $\cdots$ & 0 \\
\hline Alondra 46 & $\ldots$ & $\cdots$ & $\cdots$ & $\cdots$ & $\cdots$ & 0 & $\mathbf{t R}$ & $\cdots$ & 0 \\
\hline OC 816 & $\ldots$ & $\cdots$ & $\ldots$ & $\cdots$ & $\ldots$ & $30 s$ & t & $\ldots$ & 0 \\
\hline OC 819 & $\ldots$ & $\ldots$ & $\ldots$ & $\ldots$ & $\ldots$ & $10 \mathrm{~s}$ & 305 & $\cdots$ & 0 \\
\hline MS 7878 & $\ldots$ & $\cdots$ & $\cdots$ & $\cdots$ & $\ldots$ & $10 \mathrm{~S}$ & $t$ & $\cdots$ & 0 \\
\hline GD 798 & $\cdots$ & $\ldots$ & $\cdots$ & ... & $\cdots$ & $\cdots$ & $\mathrm{t}$ & $\cdots$ & $\cdots$ \\
\hline GD 799 & $\cdots$ & $\cdots$ & $\cdots$ & $\cdots$ & $\cdots$ & $\cdots$ & $\mathrm{t}$ & $\cdots$ & 0 \\
\hline PF 79606 & $\cdots$ & $\cdots$ & $\cdots$ & $\ldots$ & $\cdots$ & IOMR & $\mathrm{t}$ & ... & 0 \\
\hline Taiamā & $\cdots$ & $\cdots$ & $\cdots$ & $\ldots$ & $\cdots$ & $\cdots$ & $\cdots$ & $\cdots$ & $\cdots$ \\
\hline Ld 7815 & $\cdots$ & $\cdots$ & $5 s$ & $20 \mathrm{~S}$ & 0 & $\cdots$ & $\cdots$ & $\cdots$ & $\ldots$ \\
\hline OC 8112 & $\cdots$ & $\cdots$ & 0 & 0 & 0 & $\cdots$ & $\cdots$ & $\cdots$ & $\cdots$ \\
\hline R $30147-77$ & $\cdots$ & $\cdots$ & $5 R$ & $5 S$ & 0 & $\cdots$ & ... & $\cdots$ & $\cdots$ \\
\hline
\end{tabular}

$\mathrm{S}=$ suscetfvel; $\mathrm{M}=$ moderadamente; $\mathrm{R}=$ resistente; $\mathrm{t}=$ traço. 
QUADRO 3. Dados médios (Escala de Cobb modificada) das reaçōes a ferrugem da folha (Puccinia recondita) obtidos pelos cultivares avaliados cm 1981, 1982 c 1983 nas locnlidades de Maracaf, Sño Jose das Laranjeirals, Pedrinhas Paulista c Palmital, nos ensaios de cultivares suscetiveis ao $\mathrm{Al}^{3+}$

\begin{tabular}{|c|c|c|c|c|c|c|c|c|c|}
\hline \multirow[b]{2}{*}{ Cultivares } & \multicolumn{2}{|c|}{$19 \times 1$} & \multicolumn{3}{|c|}{1982} & \multicolumn{4}{|c|}{1983} \\
\hline & $\begin{array}{l}\text { São Josê das } \\
\text { Laranjeiras }\end{array}$ & Maracal & Maracal & $\begin{array}{l}\text { Pedrinhas } \\
\text { Paulista }\end{array}$ & Palmital & $\begin{array}{l}\text { São José das } \\
\text { Laranjeiras }\end{array}$ & Maracaí & $\begin{array}{c}\text { Pedrinhas } \\
\text { Paulista }\end{array}$ & Palmital \\
\hline Anahuac & $5 S$ & $10 \mathrm{~s}$ & $\ldots$ & $\ldots$ & $\ldots$ & 10MS & $20 S$ & 0 & $5 \mathrm{MR}$ \\
\hline BH 1146 & $10 \mathrm{~s}$ & $30 \mathrm{~S}$ & $20 \mathrm{~s}$ & $30 \mathrm{~s}$ & $30 \mathrm{~S}$ & $10 \mathrm{~S}$ & $20 \mathrm{~S}$ & $30 \mathrm{~s}$ & $20 S$ \\
\hline Cocoraque & 5S & $t$ & $\ldots$ & $\ldots$ & $\ldots$ & $\ldots$ & $\ldots$ & $\ldots$ & $\ldots$ \\
\hline Candeias & $5 \mathrm{~S}$ & $t$ & $5 S$ & 0 & $10 \mathrm{MS}$ & $\ldots$ & $\cdots$ & $\ldots$ & $\ldots$ \\
\hline IAPAR 3 & 0 & 0 & $\ldots$ & $\cdots$ & $\ldots$ & $\ldots$ & $\ldots$ & $\ldots$ & $\ldots$ \\
\hline IAC 15 & $15 \$$ & $20 S$ & 0 & $10 \mathrm{~S}$ & $20 S$ & $\ldots$ & $\cdots$ & $\ldots$ & $\cdots$ \\
\hline Klein Toledo & $t$ & 5S & $\ldots$ & $\ldots$ & $\ldots$ & $\ldots$ & $\ldots$ & $\ldots$ & $\ldots$ \\
\hline Diamante INTA & $20 s$ & $50 s$ & $\cdots$ & $\ldots$ & $\ldots$ & $\ldots$ & $\ldots$ & $\ldots$ & $\cdots$ \\
\hline IAPAR 6 & $10 S$ & 105 & $10 \mathrm{~s}$ & $20 S$ & $30 \mathrm{~s}$ & $\ldots$ & $\ldots$ & $\cdots$ & $\cdots$ \\
\hline Jandaia & 0 & 0 & $\cdots$ & $\cdots$ & $\ldots$ & $\cdots$ & $\ldots$ & $m$ & $\cdots$ \\
\hline OC-731070 & $5 S$ & 5S & $\ldots$ & $\ldots$ & $\cdots$ & $\cdots$ & $\cdots$ & $\cdots$ & $\cdots$ \\
\hline Pavão & $10 S$ & 305 & $10 S$ & $\mathbf{t}$ & $30 \mathrm{~S}$ & $\cdots$ & $\cdots$ & $\cdots$ & $\cdots$ \\
\hline OC 804 & $\mathbf{t}$ & $10 \mathrm{~S}$ & $\cdots$ & $\cdots$ & $\ldots$ & $\cdots$ & $\cdots$ & $\cdots$ & $\cdots$ \\
\hline OC 807 & 0 & $t$ & $\ldots$ & $\cdots$ & $\ldots$ & $\cdots$ & $\ldots$ & $\cdots$ & $\ldots$ \\
\hline OC 808 & 0 & $5 S$ & $\ldots$ & $\ldots$ & $\ldots$ & $\ldots$ & $\ldots$ & $\ldots$ & $\ldots$ \\
\hline Sparmow"S" & $25 \mathrm{MS}$ & 10MS & 0 & 0 & $30 \mathrm{~S}$ & ss & $30 \mathrm{~s}$ & 5MS & loMS \\
\hline MS 80-1 & $20 \mathrm{~s}$ & $30 s$ & $\ldots$ & $\ldots$ & $\ldots$ & $\ldots$ & $\cdots$ & ... & $\cdots$ \\
\hline Ld 7821 & $10 S$ & $30 \mathrm{MS}$ & 0 & 0 & 5s & $\ldots$ & $\ldots$ & $\cdots$ & $\ldots$ \\
\hline 1A 78103 & $\ldots$ & $\ldots$ & 0 & 0 & $30 \mathrm{~S}$ & $\ldots$ & $\ldots$ & $\ldots$ & $\cdots$ \\
\hline IAC 23 & $\ldots$ & $\cdots$ & ss & $10 \mathrm{~S}$ & $20 S$ & $\ldots$ & $10 \mathrm{~s}$ & $20 S$ & $10 \mathrm{MS}$ \\
\hline IAC 24 & $\ldots$ & $\cdots$ & sS & $20 \mathrm{~S}$ & $50 s$ & ts & $10 \mathrm{~s}$ & $20 S$ & $40 S$ \\
\hline INDUSEM 3 & $\ldots$ & $\ldots$ & $20 \mathrm{~s}$ & $20 \mathrm{MS}$ & $40 S$ & 5S & $20 \mathrm{~S}$ & $40 \mathrm{~S}$ & $40 S$ \\
\hline IOC 813 & $\ldots$ & $\ldots$ & 0 & 0 & 0 & t & $\mathrm{t}$ & $5 \mathrm{~S}$ & $20 \mathrm{MS}$ \\
\hline MS 7810 & $\cdots$ & $m$ & 0 & $20 \mathrm{~S}$ & 105 & $10 S$ & $10 S$ & $20 \mathrm{MS}$ & $30 S$ \\
\hline OCEPAR 6 & $\ldots$ & $\ldots$ & $\cdots$ & $\ldots$ & $\ldots$ & $10 S$ & $5 \mathrm{MS}$ & $10 \mathrm{MS}$ & $20 \mathrm{MS}$ \\
\hline OC 8120 & ... & $\ldots$ & 105 & $5 S$ & $10 S$ & los & $20 s$ & $40 S$ & $10 \mathrm{~S}$ \\
\hline Alondra 46 & $\ldots$ & $\ldots$ & $\ldots$ & $\ldots$ & $\ldots$ & $30 S$ & $30 \mathrm{~S}$ & $30 S$ & $40 S$ \\
\hline OC 816 & $\ldots$ & $\ldots$ & $\ldots$ & $\ldots$ & ... & 10MS & $20 \mathrm{~S}$ & $30 S$ & $50 S$ \\
\hline OC 819 & $\ldots$ & ... & $\cdots$ & $\ldots$ & $\ldots$ & $\ldots$ & $20 s$ & $20 S$ & $20 \mathrm{MS}$ \\
\hline MS 7878 & $\cdots$ & $\cdots$ & $\cdots$ & $m$ & $\cdots$ & 105 & SMS & $20 s$ & $\ldots$ \\
\hline GO 798 & $\cdots$ & $\cdots$ & $\cdots$ & $\ldots$ & $\ldots$ & $\cdots$ & $10 \mathrm{~S}$ & $\cdots$ & $\ldots$ \\
\hline GD 799 & $\ldots$ & $\ldots$ & $\cdots$ & $\cdots$ & $\ldots$ & $20 \mathrm{MS}$ & $10 S$ & $20 S$ & $20 \mathrm{MS}$ \\
\hline PF 79606 & $\cdots$ & $\cdots$ & $\cdots$ & $\cdots$ & $\cdots$ & $20 S$ & $30 S$ & $50 \mathrm{~s}$ & $40 S$ \\
\hline Taiamã & $\ldots$ & $\ldots$ & $\cdots$ & $\ldots$ & $\ldots$ & $\ldots$ & $\ldots$ & $\ldots$ & $\cdots$ \\
\hline Ld 7815 & $\ldots$ & $\cdots$ & $\mathbf{t R}$ & 0 & 0 & $\ldots$ & $\cdots$ & $\cdots$ & $\cdots$ \\
\hline OC 8112 & $\ldots$ & $\cdots$ & 5MS & 10 & $30 \mathrm{MS}$ & $\ldots$ & $\cdots$ & $\cdots$ & $\cdots$ \\
\hline R $30147-77$ & $\ldots$ & $\cdots$ & $20 S$ & 10MS & $40 \mathrm{MS}$ & $\ldots$ & $\cdots$ & $\cdots$ & $\cdots$ \\
\hline
\end{tabular}

$S=$ suscetivel; $M=$ moderadamente; $R=$ resistente; $t=$ traço.

\section{Ensaio de cultivares de trigo para solos com aluminio tóxico}

A maioria dos solos dos experimentos apresenta baixa concentração de $\mathrm{Al}^{3+}$ na camada arável, em vista das correçōes feitas pelos agricultores, mas grande área do Vale do Paranapanema apresenta solos ácidos, sendo essa acidez encontrada abaixo da camada arável, pois as correções só têm atingido, no máximo, uma profundidade de $0,30 \mathrm{~m}$.

No quadro 5, encontra-se o rendimento médio de gräos de trigo nos cultivares com tolerância ao alumínio tóxico: verifica-se que o melhor ano para a produção foi 1981, enquanto em 1982 e 1983 se obtiveram as menores produções, talvez pelas mesmas causas anteriores. 
QUADRO 4. Dados médios (Escala de Cobb modificada) das reaçöes às manchas foliares (Helminthosporium sativum) obtidos pelos cultivares avaliados em 1981 e 1983 nas localidades de Maracaí, São José das Laranjeiras, Pedrinhas Paulista e Palmital, nos ensaios de cultivares suscetiveis ao $\mathrm{Al}^{3}+$

\begin{tabular}{|c|c|c|c|c|c|}
\hline \multirow{2}{*}{ Cultivares } & \multirow{2}{*}{1981} & \multicolumn{4}{|c|}{1983} \\
\hline & & $\begin{array}{c}\text { São José das } \\
\text { Laranjeiras }\end{array}$ & Maracaí & $\begin{array}{l}\text { Pedrinhas } \\
\text { Paulista }\end{array}$ & Palmital \\
\hline Anahuac & 10 & 10 & 40 & 50 & 30 \\
\hline BH 1146 & 5 & 10 & 30 & 30 & 20 \\
\hline Cocoraque & 10 & $\cdots$ & $\ldots$ & $\ldots$ & ... \\
\hline Candeias & 10 & $\ldots$ & $\ldots$ & $\ldots$ & $\ldots$ \\
\hline IAPAR 3 & 5 & $\ldots$ & $\ldots$ & $\ldots$ & $\ldots$ \\
\hline IAC 15 & 10 & $\cdots$ & $\ldots$ & $\ldots$ & ... \\
\hline Klein Toledo & 10 & $\cdots$ & $\ldots$ & $\cdots$ & ... \\
\hline Diamante INTA & 20 & $\ldots$ & $\ldots$ & $\ldots$ & $\ldots$ \\
\hline IAPAR 6 & 5 & $\ldots$ & $\ldots$ & $\ldots$ & $\cdots$ \\
\hline Jandaia & 10 & $\cdots$ & $\ldots$ & $\ldots$ & $\ldots$ \\
\hline OC 731070 & 15 & $\ldots$ & $\ldots$ & $\cdots$ & $\ldots$ \\
\hline Pavāo & 5 & $\cdots$ & $\ldots$ & $\cdots$ & $\cdots$ \\
\hline OC 804 & 15 & $\cdots$ & $\ldots$ & $\cdots$ & $\ldots$ \\
\hline OC 807 & 10 & $\ldots$ & $\ldots$ & $\ldots$ & $\cdots$ \\
\hline OC 808 & 10 & $\cdots$ & $\ldots$ & $\cdots$ & $\cdots$ \\
\hline Sparrow "S" & 5 & 10 & 30 & 50 & 30 \\
\hline MS 80-1 & 10 & $\cdots$ & $\ldots$ & $\ldots$ & $\ldots$ \\
\hline Ld 7821 & 20 & $\ldots$ & $\ldots$ & $\ldots$ & $\ldots$ \\
\hline IA 78103 & $\ldots$ & $\ldots$ & $\ldots$ & $\ldots$ & ... \\
\hline IAC 23 & $\ldots$ & 10 & 20 & 30 & 20 \\
\hline IAC 24 & $\ldots$ & 10 & 40 & 60 & 30 \\
\hline INDUSEM 3 & $\ldots$ & 20 & 40 & 40 & 30 \\
\hline IOC 813 & $\ldots$ & 10 & 30 & 60 & 30 \\
\hline MS 7810 & $\ldots$ & 20 & 20 & 50 & 30 \\
\hline OCEPAR 6 & $\ldots$ & 20 & 30 & 40 & 30 \\
\hline OC 8120 & $\ldots$ & 10 & 30 & 40 & 30 \\
\hline Alondra 46 & $\ldots$ & 20 & 30 & 40 & 20 \\
\hline OC 816 & $\ldots$ & 20 & 30 & 50 & 30 \\
\hline OC 819 & $\ldots$ & 20 & 20 & 30 & 20 \\
\hline MS 7878 & $\ldots$ & 20 & 40 & 60 & 30 \\
\hline GD 798 & $\ldots$ & $\ldots$ & 30 & $\ldots$ & ... \\
\hline GD 799 & $\cdots$ & 20 & 30 & 30 & 30 \\
\hline PF 79606 & $\ldots$ & 10 & 30 & 40 & 20 \\
\hline Taiamã & $\ldots$ & $\ldots$ & $\ldots$ & $\ldots$ & $\ldots$ \\
\hline Ld 7815 & $\ldots$ & $\ldots$ & $\ldots$ & $\ldots$ & $\cdots$ \\
\hline OC 8112 & $\ldots$ & $\ldots$ & $\ldots$ & $\ldots$ & $\ldots$ \\
\hline R 30147-77 & $\cdots$ & $\ldots$ & $\ldots$ & $\ldots$ & $\ldots$ \\
\hline
\end{tabular}




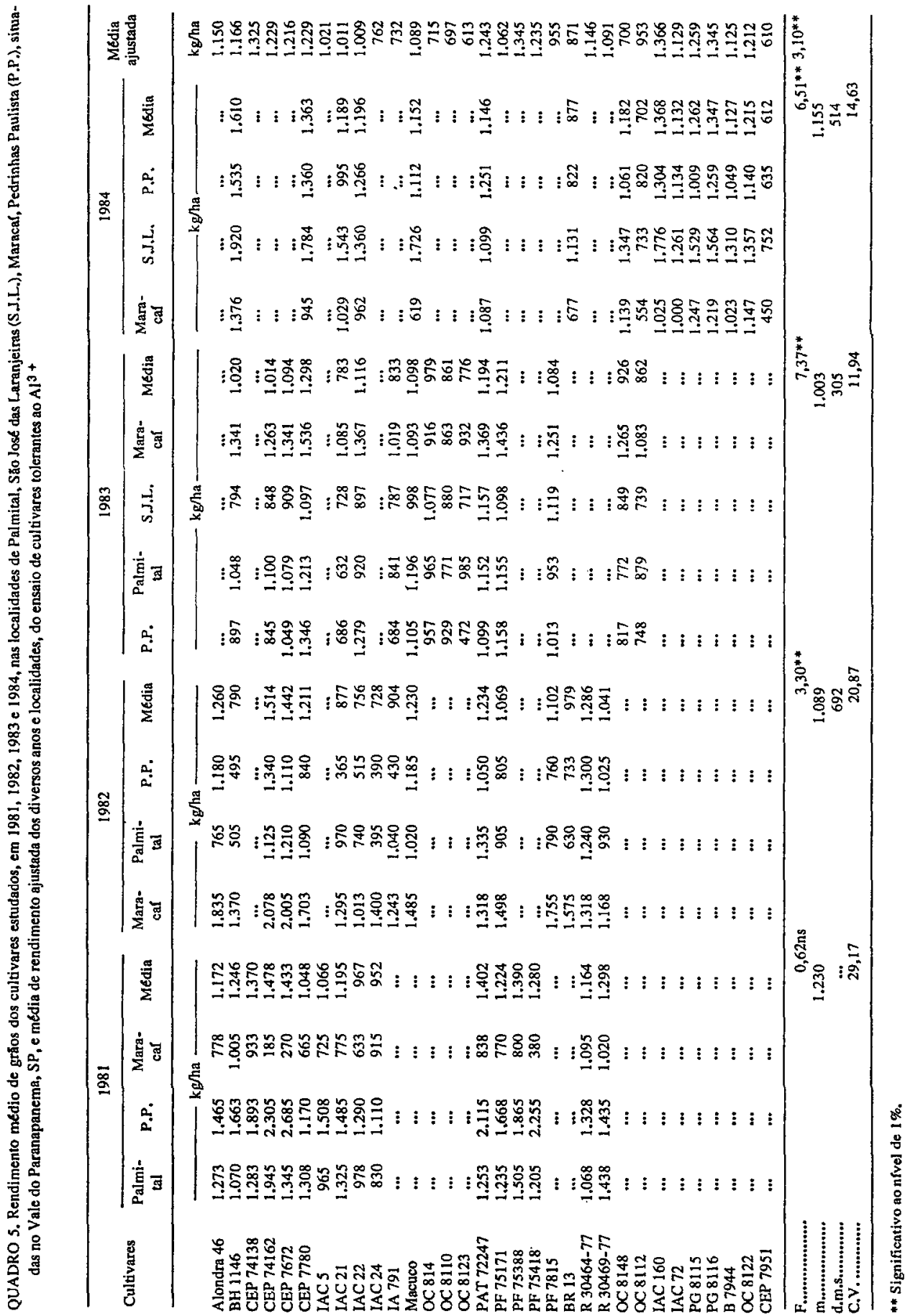


Nesses ensaios, foram considerados como tratamentos comuns os cultivares BH 1146, CEP 7780, IAC 21, IAC 22 e PAT 72247. O resultado da análise estatística apresentou $F=3,10^{\star *}$, para os tratamentos e não revelou significância na interação entre tratamentos $x$ experimentos mostrando a não-ocorrência de comportamento diferenciado dos cultivares de acordo com o ano e local do experimento.

Entre os cultivares estudados no decorrer do quadriênio, destacaram-se o PAT 72247, CEP 7780 e BH 1146, e em um segundo plano, em três anos de experimentos, 'CEP 74162 e. 'CEP 7672'. Em 1984, que apresentou o menor indice médio de precipitação no quadriênio, pode-se dar um destaque especial aos cultivares IAC 160, PG 8115, PG 8116 e OC 8122, além dos cultivares BH 1146 e CEP 7672, como sendo provavelmente os que melhor se adaptem às condições de seca mais intensa.

No quadro 6, encontra-se a reação dos cultivares de trigo à ferrugem do colmo: novamente, a maior incidência do patógeno recaiu sobre o 'BH 1146' nos três anos, chegando a atingir, em 1982, $80 \%$ na localidade de Pedrinhas Paulista. Os demais cultivares, com exceção de CEP 74138, CEP 7780 e R 30464-77, apresentaram reações que, em condição de campo, podem ser consideradas como moderadamente resistentes. Com relaçāo à ferrugem da folha, cujas reaçōes se encontram no quadro 7, os cultivares, de modo geral, comportaram-se como suscetiveis, apresentando mais intensidade do que para a ferrugem do colmo: destacaram-se com menor indice de infecção: 'CEP 7672', 'CEP 7780', 'Macuco', 'OC 813' e 'PAT 72247.

O quadro 8 revela que a ocorrência da helmintosporiose está correlacionada com o periodo de exposição da planta à umidade, e esta com a inoculação do patógeno, pois, em 1981, a incidência da moléstia não foi relevante para causar dano à produção, enquanto nos anos seguintes houve alta ocorrência em todos os cultivares.

\section{Ensaios de cultivares de trigo comerciais}

No quadro 9 , o valor de $F=1,59^{* \star}$ demonstra diferença significativa entre os tratamentos comuns: Alondra 46, BH 1146, IAC 5, IAC 17, IAC 18 e Paraguay 281, ocorrendo o mesmo para a interação tratamentos comuns $x$ experimentos, indicando que os cultivares comuns se comportaram diferencialmente durante o período estudado. Fatores como a variação climática e ocorrência de moléstias contribuíram para essa condição. Um exemplo refere-se ao 'BH 1146', que, nos dois anos de menores precipitações, 1981 e 1984, apresentou a melhor média, considerando-se os tratamentos comuns. 
QUADRO 6. Dados médios (Escala de Cobb modificada) das reaçōes à ferrugem do colmo (Puccinia graminis tritici) obtidos pelos cultivares avaliados em 1981, 1982 e 1983, nas localidades de Maracai, Palmital, Pedrinhas Paulista e São José das Laranjeiras, nos ensaios de cultivares tolerantes ao $\mathrm{Al}^{3+}$

\begin{tabular}{|c|c|c|c|c|c|c|c|}
\hline \multirow[b]{2}{*}{ Cultivares } & \multirow{2}{*}{$\frac{1981}{\text { Maracaí }}$} & \multicolumn{3}{|c|}{1982} & \multicolumn{3}{|c|}{1983} \\
\hline & & Maracaí & Palmital & $\begin{array}{l}\text { Pedrinhas } \\
\text { Paulista }\end{array}$ & $\begin{array}{l}\text { Pedrinhas } \\
\text { Paulista }\end{array}$ & Palmital & $\begin{array}{l}\text { Säo José das } \\
\text { Laranjeiras }\end{array}$ \\
\hline Alondra 46 & 0 & 0 & 0 & $\mathrm{tR}$ & $\ldots$ & $\ldots$ & $\ldots$ \\
\hline BH 1146 & $25 \mathrm{~S}$ & $30 \mathrm{~s}$ & $60 \mathrm{~S}$ & $80 S$ & 0 & $10 \mathrm{~S}$ & $20 \mathrm{~S}$ \\
\hline CEP 74138 & $5 S$ & $20 S$ & $20 \mathrm{MS}$ & $40 S$ & $\ldots$ & $\ldots$ & $\ldots$ \\
\hline CEP 74162 & $10 S$ & 0 & $5 \mathrm{~S}$ & $20 \mathrm{MR}$ & $\ldots$ & $\ldots$ & $\ldots$ \\
\hline CEP 7672 & $10 S$ & 0 & $\mathrm{t}$ & 20MR & 0 & 10MR & $10 S$ \\
\hline CEP 7780 & 0 & $10 \mathrm{~S}$ & $40 S$ & $60 S$ & 0 & 0 & $10 \mathrm{~S}$ \\
\hline IAC 5 & $5 S$ & $\ldots$ & $\ldots$ & $\ldots$ & $\ldots$ & $\cdots$ & $\ldots$ \\
\hline IAC 21 & $5 S$ & 0 & $20 \mathrm{~S}$ & $20 \mathrm{~S}$ & 0 & $10 S$ & $20 \mathrm{~S}$ \\
\hline IAC 22 & 0 & 0 & $5 S$ & $10 \mathrm{MR}$ & $c$ & 0 & $10 \mathrm{~S}$ \\
\hline IAC 24 & 0 & 0 & $10 \mathrm{~s}$ & $20 \mathrm{MR}$ & $\ldots$ & $\ldots$ & $\ldots$ \\
\hline IA 791 & $\ldots$ & 0 & $10 \mathrm{~S}$ & $20 S$ & 0 & 0 & $10 \mathrm{~S}$ \\
\hline Macuco & $\ldots$ & 0 & 0 & 0 & 0 & 0 & $5 \mathrm{MS}$ \\
\hline OC 813 & ... & 0 & 0 & 0 & 0 & 0 & $\mathrm{t} S$ \\
\hline OC 814 & ... & 0 & 0 & $5 \mathrm{MS}$ & 0 & 0 & 0 \\
\hline OC 8110 & $\ldots$ & 0 & 5S & 10MR & 0 & 0 & tS \\
\hline OC 8123 & $\ldots$ & 0 & 0 & 0 & 0 & 0 & 0 \\
\hline PAT 72247 & 0 & 0 & 0 & 0 & 0 & 0 & $20 \mathrm{~S}$ \\
\hline PF 75171 & 0 & 0 & $\mathrm{t}$ & $5 \mathrm{R}$ & 0 & 0 & $10 \mathrm{~S}$ \\
\hline PF 75388 & $t$ & 0 & $t$ & $20 S$ & $\cdots$ & ... & $\ldots$ \\
\hline PF 75418 & $5 S$ & $10 S$ & $5 \mathrm{MS}$ & $30 \mathrm{~S}$ & $\cdots$ & ... & $\ldots$ \\
\hline PF 7815 & $\ldots$ & 0 & 0 & $5 S$ & 0 & 0 & 10MR \\
\hline PF 782027 & $\ldots$ & 0 & 0 & $10 \mathrm{~S}$ & $\ldots$ & $\cdots$ & $\ldots$ \\
\hline R 30464-77 & 0 & 0 & $t$ & $5 S$ & $\cdots$ & ... & ... \\
\hline R 30469-77 & 5 & 0 & $20 S$ & $60 \mathrm{~S}$ & $\cdots$ & ... & ... \\
\hline OC 8148 & $\cdots$ & ... & $\cdots$ & $\cdots$ & 0 & 0 & $\ldots$ \\
\hline OC 8112 & $\ldots$ & $\ldots$ & $\ldots$ & ... & 0 & 0 & $\mathbf{t} \mathbf{S}$ \\
\hline
\end{tabular}

$\mathrm{S}=$ suscetível; $\mathrm{M}=$ moderadamente; $\mathrm{R}=$ resistente; $\mathrm{t}=$ traço.

Considerando a reação à ferrugem do colmo - Quadro 10 - o 'BH 1146', em 1981 e 1982, foi altamente suscetível, com nota de até $90 \mathrm{~S}$, em 1982, nas localidades de São José das Laranjeiras e Pedrinhas Paulista, enquanto o.'Alondra $46^{\prime}$ apresentou, nesses locais, notas zero e $5 R$ respectivamente, permanecendo como resistente às raças do patógeno ocorrentes na regiāo. $O$ reflexo dessa diferenciaçāo de reaçōes pode estar expresso, pelo menos em parte em 1982, na produção de gräos de $1.232 \mathrm{~kg} / \mathrm{ha}$ para o cultivar Alondra 46 e de $764 \mathrm{~kg} / \mathrm{ha}$ para o BH 1146. 
QUADRO 7. Dados médios (Escala de Cobb modificada) das reaçōes à ferrugem da folha (Puccinia recondita) obtidos pelos cultivares avaliados em 1981, 1982 e 1983, nas localidades de Maracaí, Palmital, Pedrinhas Paulista e São José das Laranjeiras, nos ensaios de cultivares tolerantes ao $\mathrm{Al}^{3}+$

\begin{tabular}{|c|c|c|c|c|c|c|c|}
\hline \multirow{2}{*}{ Cultivares } & \multirow{2}{*}{$\begin{array}{c}1981 \\
\text { Maracaí }\end{array}$} & \multicolumn{3}{|c|}{1982} & \multicolumn{3}{|c|}{1983} \\
\hline & & Maracaí & Palmital & $\begin{array}{l}\text { Pedrinhas } \\
\text { Paulista }\end{array}$ & $\begin{array}{l}\text { Pedrinhas } \\
\text { Paulista }\end{array}$ & Palmital & $\begin{array}{l}\text { São José das } \\
\text { Laranjeiras }\end{array}$ \\
\hline Alondra 46 & 0 & 0 & $50 \mathrm{~S}$ & $\mathrm{tS}$ & $\ldots$ & $\ldots$ & $\ldots$ \\
\hline BH 1146 & $20 S$ & $10 S$ & $30 S$ & $10 S$ & $30 S$ & $20 S$ & $20 S$ \\
\hline CEP 74138 & $\mathbf{t}$ & 0 & $10 S$ & $20 \mathrm{~S}$ & $\ldots$ & $\ldots$ & $\ldots$ \\
\hline CEP 74162 & 0 & 0 & 0 & 0 & $\ldots$ & $\ldots$ & $\ldots$ \\
\hline CEP 7672 & $5 S$ & 0 & 0 & 0 & 0 & $10 \mathrm{~S}$ & $5 \mathrm{MS}$ \\
\hline CEP 7780 & 0 & 0 & $30 \mathrm{~S}$ & $20 S$ & 0 & SMR & $5 S$ \\
\hline IAC 5 & $20 S$ & $\ldots$ & $\ldots$ & 0 & $\ldots$ & $\ldots$ & $\ldots$ \\
\hline IAC 21 & $15 S$ & 5 & $30 s$ & $10 \mathrm{~S}$ & $20 S$ & $40 \mathrm{~S}$ & $10 \mathrm{~S}$ \\
\hline IAC 22 & $20 S$ & 5 & $40 \mathrm{~S}$ & $20 S$ & $30 S$ & $20 S$ & $10 S$ \\
\hline IAC 24 & $10 \mathrm{MS}$ & 0 & $40 S$ & $10 \mathrm{~S}$ & ... & $\ldots$ & $\ldots$ \\
\hline IA 791 & $\ldots$ & 0 & $40 S$ & 5 & $20 s$ & $30 s$ & $20 S$ \\
\hline Macuco & $\ldots$ & 0 & 0 & 0 & 0 & 0 & $5 S$ \\
\hline OC 813 & ... & 0 & 0 & 0 & 0 & 0 & 5MR \\
\hline OC 814 & $\ldots$ & 0 & $20 S$ & 0 & $20 S$ & $30 \mathrm{MS}$ & $40 S$ \\
\hline OC 8110 & $\ldots$ & 0 & $30 S$ & 0 & $20 S$ & $40 S$ & $\ldots$ \\
\hline OC 8123 & $\ldots$ & 0 & 0 & 0 & 0 & 20MS & $20 S$ \\
\hline PAT 72247 & $5 S$ & 0 & 0 & 0 & 0 & $10 S$ & $10 S$ \\
\hline PF 75171 & $5 S$ & 0 & $10 \mathrm{MS}$ & $20 S$ & $10 S$ & $40 S$ & $10 S$ \\
\hline PF 75388 & $10 S$ & 0 & $20 S$ & $10 S$ & $\ldots$ & ... & $\ldots$ \\
\hline PF 75418 & $20 S$ & $10 \mathrm{MS}$ & $30 S$ & $10 S$ & ... & $\ldots$ & $\ldots$ \\
\hline PF 7815 & $\ldots$ & $5 \mathrm{MS}$ & $30 \mathrm{MS}$ & $20 \mathrm{MS}$ & $30 \mathrm{MS}$ & $30 S$ & $20 S$ \\
\hline PF 782027 & $\ldots$ & $10 S$ & $30 S$ & $10 S$ & $\ldots$ & $\ldots$ & $\ldots$ \\
\hline R 30464-77 & $10 S$ & 0 & $40 S$ & 5MS & $\ldots$ & $\ldots$ & $\ldots$ \\
\hline R 30469-77 & $10 S$ & 0 & $30 s$ & $20 S$ & $\ldots$ & $\ldots$ & $\ldots$ \\
\hline OC 8148 & $\ldots$ & $\ldots$ & $\ldots$ & $\ldots$ & 0 & $20 s$ & 0 \\
\hline CC 8112 & $\ldots$ & $\ldots$ & $\ldots$ & $\ldots$ & $40 S$ & $60 S$ & $40 S$ \\
\hline
\end{tabular}

$S=$ stuscetivel; $M=$ moderadamente; $R=$ resistente; $t=$ traço.

Outro cultivar que se destacou é o Paraguay 281: apresentou uma produção decrescente ao longo do estudo, iniciando o período com uma produção média de $1.391 \mathrm{~kg} / \mathrm{ha}$ em 1981 e decrescendo para 678,929 e $758 \mathrm{~kg} / \mathrm{ha}$, de acordo com os anos. Isso provavelmente se deva à sua exigência sobretudo no que se refere à disponibilidade de água no solo, associada às reações de susceti- 
bilidade às doenças, principalmente à ferrugem do colmo, que, embora se tenha manifestado mais tardiamente nos três últimos anos de estudo, pode ter sido mais drástica para ele, visto apresentar ciclo tardio. Também, nesse caso, o complexo de manchas foliares pode ter sido fator importante na redução de produtividade, pois mesmo nos anos de altas precipitações, não se verificou aumento.

QUADRO 8. Dados médios (Escala de Cobb modificada) das reações às manchias foliares (Helminthosporium sativum) obtidos pelos cultivares em 1981, 1982 e 1983, nas localidades de Maracaí, Palmital, Pedrinhas Paulista e São José das Laranjeiras, nos ensaios de cultivares tolerantes ao $\mathrm{Al}^{3}+$

\begin{tabular}{lcccccc}
\hline & 1981 & & 1982 & & \multicolumn{3}{c}{1983} \\
\cline { 2 - 2 } \cline { 6 - 7 } Cultivares & Maracaí & Palmital & $\begin{array}{c}\text { Pedrinhas } \\
\text { Paulista }\end{array}$ & Palmital & $\begin{array}{c}\text { São José das } \\
\text { Laranjeiras }\end{array}$ \\
\hline
\end{tabular}

\begin{tabular}{|c|c|c|c|c|c|}
\hline Alondra 46 & 20 & 40 & $\cdots$ & $\cdots$ & ... \\
\hline BH 1146 & 15 & 40 & 30 & 30 & 20 \\
\hline CEP 74138 & 10 & 20 & ... & $\ldots$ & ... \\
\hline CEP 74162 & 5 & 20 & ... & $\ldots$ & ... \\
\hline CEP 7672 & 5 & 30 & 40 & 30 & 20 \\
\hline CEP 7780 & 15 & 20 & 50 & 30 & 10 \\
\hline IAC 5 & 10 & $\ldots$ & $\ldots$ & $\ldots$ & ... \\
\hline IAC 21 & 10 & 40 & 40 & 40 & 20 \\
\hline IAC 22 & 15 & 20 & 40 & 40 & 20 \\
\hline IAC 24 & 15 & 40 & $\ldots$ & $\ldots$ & ... \\
\hline IA 791 & $\ldots$ & 30 & 50 & 20 & 10 \\
\hline Macuco & $\ldots$ & 30 & 50 & 30 & 20 \\
\hline OC 813 & $\ldots$ & 40 & 50 & 40 & 20 \\
\hline OC 814 & $\ldots$ & 50 & 50 & 30 & 20 \\
\hline OC 8110 & $\ldots$ & 40 & 50 & 40 & 20 \\
\hline OC 8123 & $\ldots$ & 30 & 50 & 40 & 30 \\
\hline PAT 72247 & 5 & 20 & 40 & 30 & 20 \\
\hline PF 75171 & 10 & 20 & 50 & 40 & 20 \\
\hline PF 75388 & 10 & 30 & ... & ... & ... \\
\hline PF 75418 & 10 & 20 & $\ldots$ & $\ldots$ & $\ldots$ \\
\hline PF 7815 & $\ldots$ & 20 & 40 & 30 & 20 \\
\hline PF 782027 & $\ldots$ & 30 & $\ldots$ & $\ldots$ & ... \\
\hline R 30464-77 & 10 & 30 & $\ldots$ & $\ldots$ & ... \\
\hline R 30469-77 & 10 & 30 & $\ldots$ & $\ldots$ & ... \\
\hline OC 8148 & $\ldots$ & $\ldots$ & 60 & 40 & 20 \\
\hline OC 8112 & $\ldots$ & $\ldots$ & 50 & 60 & 30 \\
\hline
\end{tabular}




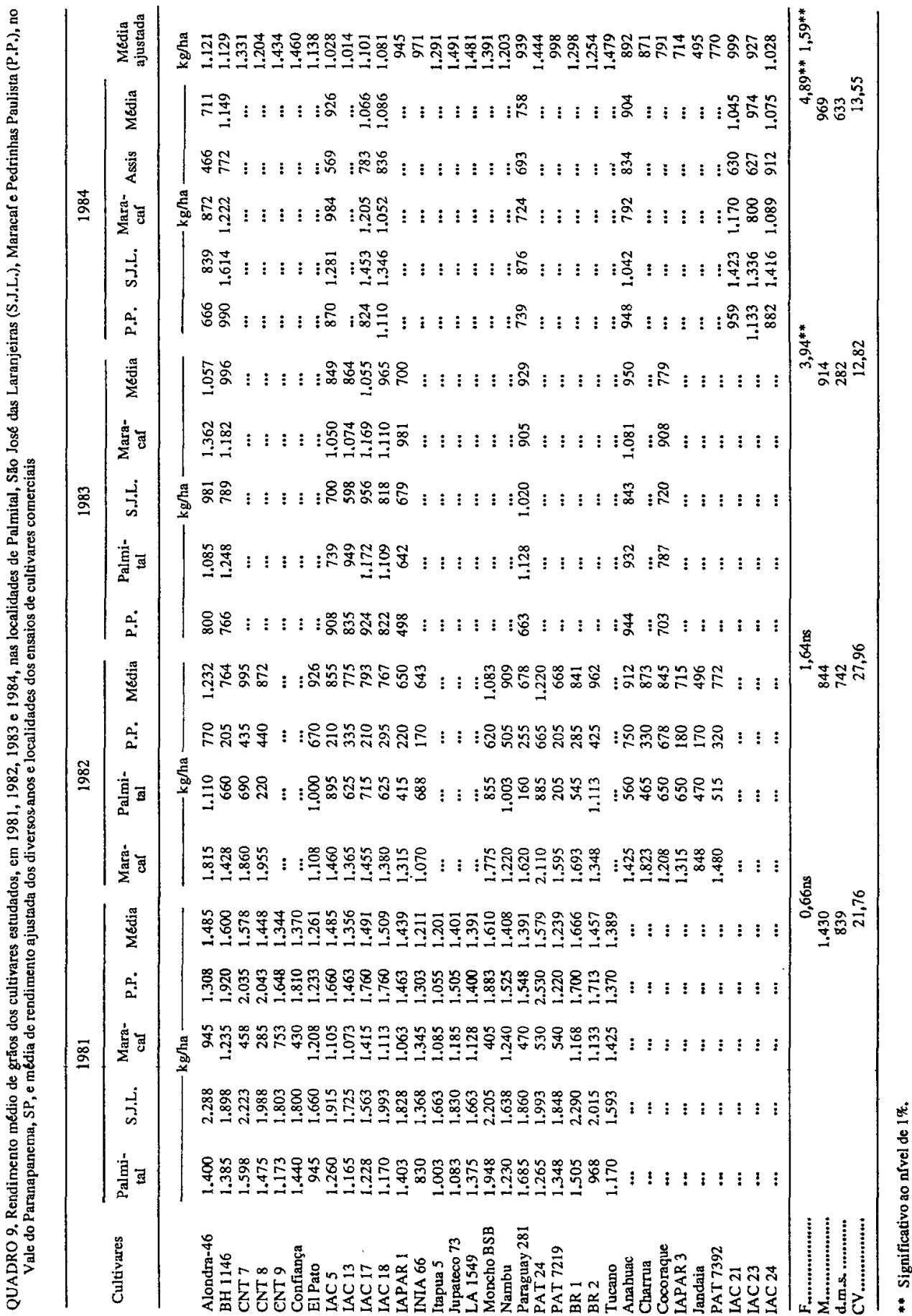


Quanto à avaliação dos genótipos estudados para resistência a Puccinia graminis tritici - Quadro 10 - podem-se destacar, em 1982 e 1983, os cultivares CNT 8, EI Pato e INIA 66, como os mais resistentes, e PAT 24, Anahuac e Cocoraque como moderadamente resistentes. Os demais não apresentaram estabilidade no período. Para a Puccinia recondita, moléstia que tem apresentado alta incidência de ataque, iniciando-se geralmente nos cultivares mais suscetiveis aos 45 dias de vida da planta, destaca-se o 'IAPAR 1 Mitacoré' como o mais resistente no periodo: Quadro 11. Em um segundo plano, aparecem os cultivares EI Pato, CNT 8 e Nambu. O 'Alondra 46', que se apresentava como resistente no inicio do período, revelou quebra dessa resistência, passando, em 1983, a altamente suscetivel.

QUADRO 10. Dados médios (Escala de Cobb modificada) das reaçóes à ferrugem do colmo (Puccinia yraminis tritici) obtidos pelos cultivares avaliados em 1981, 1982 e 1983 nas localidades de Sāo José das Laranjeiras, Maracaf, Palmital e Pedrinhas Paulista nos ensaios de cultivares comerciais

\begin{tabular}{|c|c|c|c|c|c|c|c|c|c|c|}
\hline \multirow{2}{*}{ Cultivares } & \multicolumn{2}{|c|}{1981} & \multicolumn{4}{|c|}{1982} & \multicolumn{4}{|c|}{1983} \\
\hline & $\begin{array}{l}\text { Săo Jose das } \\
\text { Laranjeiras }\end{array}$ & Maracaf & $\begin{array}{l}\text { Sāo Josê das } \\
\text { Laranjeiras }\end{array}$ & Palmital & $\begin{array}{c}\text { Pedrinhas } \\
\text { Paulista }\end{array}$ & Maracal & Maracal & $\begin{array}{l}\text { S7a Jose das } \\
\text { Laranjeiras }\end{array}$ & Palmital & $\begin{array}{c}\text { Pedrinhas } \\
\text { Paulista }\end{array}$ \\
\hline Alondra 46 & 0 & 0 & 0 & 0 & $5 R$ & 0 & $\mathbf{0}$ & $\mathbf{t S}$ & 0 & 0 \\
\hline BH 1146 & $40 S$ & SS & 905 & $70 \mathrm{~s}$ & 905 & $40 \mathrm{~S}$ & $10 S$ & $20 S$ & $10 S$ & 105 \\
\hline CNT 7 & $\mathbf{t S}$ & 0 & 0 & $\mathbf{t S}$ & $10 S$ & 0 & $\cdots$ & $\cdots$ & $\ldots$ & ... \\
\hline CNT 8 & 0 & 0 & 0 & 0 & 0 & 0 & $\cdots$ & $\cdots$ & $\ldots$ & ... \\
\hline CNT 9 & 0 & 0 & ... & $\cdots$ & $\ldots$ & $\ldots$ & $\ldots$ & $\cdots$ & $\cdots$ & $\ldots$ \\
\hline Confiança & $\mathbf{t S}$ & 0 & $\ldots$ & $\cdots$ & $\ldots$ & $\cdots$ & $\ldots$ & $\ldots$ & $\cdots$ & $\ldots$ \\
\hline E1 Pato & 0 & 0 & 0 & 0 & 0 & 0 & $\ldots$ & $\ldots$ & $\ldots$ & $\ldots$ \\
\hline IAC 5 & tS & $5 S$ & $20 S$ & $40 S$ & $40 S$ & $20 S$ & $20 S$ & 105 & 105 & $10 S$ \\
\hline IAC 13 & tS & 0 & 10MR & SR & $S R$ & 10MR & $20 S$ & $20 S$ & $20 S$ & $10 S$ \\
\hline IAC 17 & SS & 0 & $30 S$ & $40 S$ & $40 S$ & $20 S$ & $20 S$ & $10 S$ & tS & 0 \\
\hline IAC 18 & 10S & $\mathbf{t S}$ & $80 S$ & $60 S$ & $60 S$ & $30 \mathrm{~S}$ & 5S & tS & $20 S$ & $\mathbf{t S}$ \\
\hline IAPAR I & $\mathbf{t S}$ & tS & $50 S$ & $40 S$ & $40 S$ & SS & $\mathbf{t S}$ & 0 & 0 & 0 \\
\hline INIA 66 & 0 & 0 & 0 & 0 & 0 & 0 & $\cdots$ & $\cdots$ & $\cdots$ & $\ldots$ \\
\hline Itapua 5 & $\mathbf{t S}$ & ts & ... & $\cdots$ & $\cdots$ & $\cdots$ & $\cdots$ & $\cdots$ & $\cdots$ & $\cdots$ \\
\hline Jupateco & ts & 0 & ' ... & ... & $\cdots$ & $\cdots$ & $\cdots$ & $\cdots$ & $\cdots$ & $\cdots$ \\
\hline LA 1549 & SS & $\mathbf{t S}$ & ... & $\ldots$ & ... & $\ldots$ & $\cdots$ & $\cdots$ & $\ldots$ & $\ldots$ \\
\hline Moncho BSB & tS & 0 & $20 S$ & $20 S$ & $20 S$ & $10 S$ & $\ldots$ & $\cdots$ & $\ldots$ & $\ldots$ \\
\hline Nambu & tS & ts & $20 \mathrm{~S}$ & $30 \mathrm{~S}$ & $30 S$ & SS & $\ldots$ & $\ldots$ & $\ldots$ & $\cdots$ \\
\hline Paraguay 281 & $\mathbf{t S}$ & 0 & $30 S$ & $30 \mathrm{~S}$ & $30 s$ & 5S & $20 S$ & $20 S$ & $20 S$ & $10 s$ \\
\hline PAT 24 & 0 & 0 & 0 & sS & 5S & $10 \mathrm{MS}$ & $\ldots$ & ... & $\ldots$ & $\cdots$ \\
\hline PAT 7219 & 5S & SS & $50 S$ & $80 S$ & 805 & $30 S$ & $\ldots$ & ... & $\ldots$ & $\ldots$ \\
\hline BR 1 & $\mathbf{t S}$ & 0 & $20 S$ & $40 S$ & $40 S$ & $20 S$ & ... & $\ldots$ & $\cdots$ & $\cdots$ \\
\hline BR 2 & tS & tS & $5 S$ & $10 \mathrm{~S}$ & I0S & 0 & $\cdots$ & $\ldots$ & ... & $\ldots$ \\
\hline Tucano & tS & tS & $\ldots$ & $\cdots$ & $\cdots$ & $\ldots$ & $\ldots$ & $\cdots$ & $\ldots$ & $\cdots$ \\
\hline Anahusac & $\ldots$ & ... & 0 & $\mathbf{S R}$ & SR & SMR & 0 & ts & 0 & 0 \\
\hline Charrua & ...* & $\cdots$ & $40 S$ & $80 S$ & $80 S$ & 20MR & $\ldots$ & $\ldots$ & $\ldots$ & $\ldots$ \\
\hline Cocoraque & $\cdots$ & $\cdots$ & 0 & 0 & 0 & tS & 0 & $5 S$ & 0 & 0 \\
\hline IAPAR 3 & $\cdots$ & $\infty$ & 0 & $10 S$ & 10s & 0 & $\cdots$ & 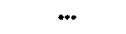 & $\cdots$ & $\cdots$ \\
\hline Jandaia & $\cdots$ & $\cdots$ & $50 S$ & $40 S$ & $40 S$ & tS & $\cdots$ & $\cdots$ & $\cdots$ & $\cdots$ \\
\hline PAT 7392 & ... & $*$ & 0 & $40 S$ & $40 S$ & tS & $\cdots$ & $\cdots$ & $\cdots$ & $\cdots$ \\
\hline IAC $21(*)$ & $\cdots$ & $\omega$ & $\cdots$ & $\cdots$ & $\cdots$ & $\cdots$ & $\cdots$ & $\cdots$ & $\cdots$ & $\cdots$ \\
\hline IAC $23\left(^{\circ}\right)$ & $\infty$ & $\cdots$ & $\cdots$ & $\cdots$ & $\cdots$ & $\cdots$ & $\cdots$ & $\cdots$ & $\cdots$ & $\cdots$ \\
\hline IAC $24\left(^{*}\right)$ & $m$ & $m$ & $\ldots$ & $\cdots$ & $\cdots$ & $\ldots$ & $\cdots$ & $\cdots$ & 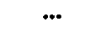 & $\cdots$ \\
\hline
\end{tabular}

$S=$ suscetfvel; $M=$ moderadamente; $R=$ resistente; $t=$ traço.

(*) Participaram apenas dos ensaios em 1984, quando nāo ocorreu a moléstia. 
QUADRO 11. Dados médios (Escala de Cobb modificada) das reaçōes à ferrugem da folha (Puccinia recondita) obtidos pelos cultivares avaliados em 1981, 1982 e 1983 nas localidades de Săo José das Lannjeiras, Maracar, Palmital e Pedrinhas Paulista nos ensaios de cultivares comerciais

\begin{tabular}{|c|c|c|c|c|c|c|c|c|c|c|}
\hline \multirow{2}{*}{ Cultivares } & \multicolumn{2}{|c|}{1981} & \multicolumn{4}{|c|}{1982} & \multicolumn{4}{|c|}{1983} \\
\hline & $\begin{array}{l}\text { São Jose das } \\
\text { Laranjeiras }\end{array}$ & Maracal & $\begin{array}{l}\text { Säo Jose das } \\
\text { Laranjeiras }\end{array}$ & Palmital & $\begin{array}{l}\text { Pedrinhas } \\
\text { Paulista }\end{array}$ & Maracar & Maracal & $\begin{array}{l}\text { Szao José das } \\
\text { Laranjeiras }\end{array}$ & Palmital & $\begin{array}{c}\text { Pedrinhas } \\
\text { Paulista }\end{array}$ \\
\hline
\end{tabular}

\begin{tabular}{|c|c|c|c|c|c|c|c|c|c|c|}
\hline Alondra 46 & 0 & 0 & 10MR & $30 \mathrm{~S}$ & 0 & $5 \mathrm{MS}$ & $20 \mathrm{~S}$ & 205 & $60 \mathrm{~S}$ & $30 s$ \\
\hline BH 1146 & $20 \mathrm{~S}$ & $20 \mathrm{~S}$ & $20 S$ & $50 \mathrm{~s}$ & 205 & $10 S$ & $20 \mathrm{~s}$ & 105 & $30 \mathrm{~S}$ & $30 \mathrm{~s}$ \\
\hline CNT 7 & $15 \mathrm{MS}$ & $20 S$ & $10 S$ & $40 S$ & $20 S$ & $20 S$ & $\ldots$ & $\ldots$ & $\ldots$ & $\ldots$ \\
\hline CNT 8 & $10 S$ & 10MS & $0-5 M R$ & $t R$ & 5MS & 0 & $\cdots$ & $\cdots$ & $\cdots$ & $\cdots$ \\
\hline CNT 9 & $20 \mathrm{~S}$ & tS & $\cdots$ & $\cdots$ & $\cdots$ & $\cdots$ & $\cdots$ & $\cdots$ & $\cdots$ & $\cdots$ \\
\hline Confiança & $20 S$ & $10 \mathrm{MS}$ & $\ldots$ & $\ldots$ & $\ldots$ & $\ldots$ & $\ldots$ & $\ldots$ & $\cdots$ & $\cdots$ \\
\hline El Pato & 0 & 5S & 0 & 0 & 0 & 0 & $\ldots$ & $\cdots$ & $\cdots$ & $\ldots$ \\
\hline IAC 5 & $20 \mathrm{~S}$ & $30 \mathrm{~s}$ & $20 S$ & sos & $20 s$ & 105 & $20 \mathrm{~s}$ & $40 S$ & $50 \mathrm{~s}$ & $30 \mathrm{~S}$ \\
\hline IAC 13 & $30 \mathrm{~S}$ & $60 \mathrm{~S}$ & $30 \mathrm{~S}$ & $40 S$ & $20 \mathrm{~S}$ & $30 \mathrm{~s}$ & $30 \mathrm{~S}$ & $30 \mathrm{~S}$ & $50 \mathrm{~s}$ & $30 \mathrm{~S}$ \\
\hline IAC 17 & $20 \mathrm{~S}$ & $40 S$ & $20 \mathrm{~S}$ & $40 \mathrm{~S}$ & $10 S$ & $20 \mathrm{~S}$ & $20 \mathrm{~S}$ & $30 \mathrm{~S}$ & $60 \mathrm{~S}$ & $30 S$ \\
\hline IAC 18 & $20 \mathrm{~S}$ & $10 \mathrm{~S}$ & $20 \mathrm{~S}$ & $40 \mathrm{~S}$ & $5 S$ & $10 S$ & $20 \mathrm{~S}$ & $10 \mathrm{~S}$ & $20 \mathrm{~s}$ & $20 \mathrm{~s}$ \\
\hline IAPAR I & 0 & 0 & 0 & 0 & 0 & 0 & 0 & 0 & 0 & 0 \\
\hline INIA 66 & $40 S$ & $30 S$ & $40 S$ & $20 S$ & $30 s$ & $30 S$ & $\cdots$ & $\cdots$ & $\cdots$ & $\ldots$ \\
\hline Itapua 5 & $30 S$ & $30 \mathrm{~S}$ & $\cdots$ & $\cdots$ & $\cdots$ & $\cdots$ & $\cdots$ & $\cdots$ & $\cdots$ & $\cdots$ \\
\hline Jupateco & $20 S$ & $20 S$ & $\cdots$ & $\cdots$ & $\cdots$ & $\cdots$ & $\cdots$ & $\cdots$ & $\cdots$ & $\cdots$ \\
\hline LA 1549 & $20 S$ & $20 S$ & $\cdots$ & $\cdots$ & $\cdots$ & $\cdots$ & $\cdots$ & $\cdots$ & $\cdots$ & $\cdots$ \\
\hline Moncho BSB & $5 \mathrm{~S}$ & 0 & 0 & $20 \mathrm{~S}$ & 0 & 5S & $\cdots$ & $\cdots$ & $\cdots$ & $\cdots$ \\
\hline Nambu & ts & $5 S$ & 0 & 0 & 0 & 0 & $\ldots$ & $\ldots$ & $\cdots$ & $\ldots$ \\
\hline Paraguay 281 & $20 \mathrm{~S}$ & $10 \mathrm{~S}$ & $10 \mathrm{~s}$ & $30 \mathrm{~S}$ & $20 S$ & 5S & $20 \mathrm{~S}$ & $50 \mathrm{~S}$ & $50 \mathrm{~S}$ & $50 \mathrm{~s}$ \\
\hline РАТ 24 & $20 \mathrm{~s}$ & $20 \mathrm{~S}$ & $20 S$ & $30 S$ & $60 \mathrm{~S}$ & $30 S$ & $\ldots$ & $\cdots$ & $\cdots$ & $\cdots$ \\
\hline PAT 7219 & 10MS & 20MS & 10MS & $30 \mathrm{MS}$ & 5MS & $20 S$ & $\cdots$ & $\cdots$ & $\cdots$ & $\cdots$ \\
\hline BR 1 & $10 \mathrm{~S}$ & $20 \mathrm{~S}$ & $5 S$ & $40 \mathrm{~S}$ & $30 \mathrm{~S}$ & $10 \mathrm{~S}$ & $\cdots$ & $\cdots$ & $\cdots$ & $\cdots$ \\
\hline BR 2 & $20 S$ & $10 S$ & $20 S$ & $20 S$ & $10 \mathrm{~S}$ & $10 S$ & $\cdots$ & $\cdots$ & $\cdots$ & $\cdots$ \\
\hline Tucano & $30 s$ & $50 \mathrm{~S}$ & $\cdots$ & $\cdots$ & $\cdots$ & $\cdots$ & $\cdots$ & $\cdots$ & … & $\dddot{m}$ \\
\hline Anahuac & $\ldots$ & $\ldots$ & 0 & 0 & tS & 0 & $5 \mathrm{MS}$ & $30 \mathrm{MS}$ & $30 \mathrm{MS}$ & 0 \\
\hline Charnia & $\ldots$ & $\cdots$ & $30 \mathrm{~S}$ & $20 \mathrm{~S}$ & $5 S$ & 0 & $\ldots$ & $\cdots$ & $\ldots$ & $\ldots$ \\
\hline Cocoraque & $\cdots$ & $\cdots$ & ts & $20 \mathrm{~S}$ & 0 & 0 & 0 & $5 S$ & 10MR & 0 \\
\hline IAPAR 3 & $\cdots$ & $\cdots$ & 0 & 0 & 0 & 0 & $\cdots$ & $\cdots$ & $\cdots$ & $\cdots$ \\
\hline Jandaia & $\cdots$ & $\cdots$ & 0 & 0 & 0 & 0 & $\cdots$ & $\cdots$ & $\cdots$ & $\cdots$ \\
\hline PAT 7392 & $\ldots$ & $\cdots$ & $\cdots$ & $10 \mathrm{MS}$ & 0 & 0 & $\cdots$ & $\cdots$ & $\cdots$ & $\cdots$ \\
\hline IAC $21\left(^{*}\right)$ & $\cdots$ & $\cdots$ & $\cdots$ & $\cdots$ & -- & $\cdots$ & $\cdots$ & $\cdots$ & $\cdots$ & $\cdots$ \\
\hline IAC $23\left(^{*}\right)$ & $\ldots$ & $\cdots$ & $\ldots$ & $\cdots$ & $\cdots$ & $\cdots$ & $\cdots$ & $\cdots$ & $\cdots$ & $\ldots$ \\
\hline IAC $24\left(^{*}\right)$ & $\cdots$ & $\cdots$ & $\cdots$ & $\cdots$ & $\ldots$ & $\cdots$ & $\ldots$ & $\cdots$ & $\cdots$ & $\cdots$ \\
\hline
\end{tabular}

$\mathrm{S}=$ suscetível; $\mathrm{M}=$ moderadamente; $\mathrm{R}=$ resistente; $\mathrm{t}=$ traço.

(*) Participaram apenas dos ensaios em 1984, quando näo ocorreu a molestia.

As manchas foliares (Helminthosporium sativum), de acordo com o quadro 12, apresentaram alta incidência; nenhum cultivar demonstrou resistência, e a ocorrência da umidade relativa do ar foi elevada, principalmente em 1982 e 1983, que, segundo LUZ (1982), contribui para que a moléstia tenha ambiente satisfatório para a sua disseminação. 
QUADRO 12. Dados medios (Escala de Cobb modificada) das reaçós a manchas foliates (Helminthosporium sativum) obtidos pelos cultivares avaliados em 1981, 1982 e 1983 nas localidades de São José das Laranjeiras, Maracal, Palmital e Pedrinhas Paulista nos ensaios de cultivares comerciais

\begin{tabular}{|c|c|c|c|c|c|c|c|c|c|}
\hline \multirow[b]{2}{*}{ Cultivares } & \multirow{2}{*}{$\frac{1981}{\text { Maracaf }}$} & \multicolumn{4}{|c|}{1982} & \multicolumn{4}{|c|}{1983} \\
\hline & & $\begin{array}{l}\text { Ş̌o Jose das } \\
\text { Laranjeiras }\end{array}$ & Palmital & $\begin{array}{c}\text { Pedrinhas } \\
\text { Paulista }\end{array}$ & Maracaf & Maracal & $\begin{array}{l}\text { São Jose das } \\
\text { Laranjeiras }\end{array}$ & Palmital & $\begin{array}{c}\text { Pedrinhas } \\
\text { Paulista }\end{array}$ \\
\hline
\end{tabular}

\begin{tabular}{|c|c|c|c|c|c|c|c|c|c|}
\hline Alondra 46 & 30 & 40 & 40 & 40 & 50 & 30 & 30 & 40 & 50 \\
\hline BH 1146 & 20 & 40 & 30 & 30 & 30 & 20 & 20 & 20 & 40 \\
\hline CNT 7 & 10 & 40 & 30 & 40 & 40 & $\ldots$ & $\cdots$ & ... & $\cdots$ \\
\hline CNT 8 & 10 & 30 & 20 & 40 & 30 & $\ldots$ & $\cdots$ & $\ldots$ & $\cdots$ \\
\hline CNT 9 & 10 & $\cdots$ & $\ldots$ & $\cdots$ & $\cdots$ & $\cdots$ & $\ldots$ & $\ldots$ & $\cdots$ \\
\hline Confiança & 20 & $\ldots$ & $\cdots$ & $\ldots$ & $\ddot{m}$ & $\cdots$ & $\cdots$ & $\cdots$ & $\cdots$ \\
\hline EI Pato & 20 & 40 & 40 & 30 & 30 & $\ldots$ & $\ldots$ & $\cdots$ & $\cdots$ \\
\hline IAC 5 & 10 & 40 & 30 & 40 & 30 & 40 & 20 & 30 & 40 \\
\hline IAC 13 & 20 & 40 & 30 & 30 & 40 & 30 & 30 & 30 & 40 \\
\hline $\mathrm{IAC} 17$ & 20 & 40 & 30 & 40 & 30 & 30 & 20 & 20 & 40 \\
\hline IAC 18 & 10 & 40 & 30 & 40 & 30 & 20 & 20 & 30 & 40 \\
\hline IAPAR 1 & 10 & 40 & 40 & 40 & 40 & 40 & 30 & 50 & 50 \\
\hline INIA 66 & 30 & 40 & 40 & 50 & 50 & $\ldots$ & $\cdots$ & $\cdots$ & $\cdots$ \\
\hline Itapua 5 & 30 & $\cdots$ & $\cdots$ & $\ldots$ & $\cdots$ & $\cdots$ & $\ldots$ & $\cdots$ & $\cdots$ \\
\hline Jupateco & 20 & $\cdots$ & $\cdots$ & $\cdots$ & $\cdots$ & $\cdots$ & $\cdots$ & $\cdots$ & $\cdots$ \\
\hline LA 1549 & 20 & $\cdots$ & $\ldots$ & $\cdots$ & $\cdots$ & $\cdots$ & ... & $\cdots$ & $\ldots$ \\
\hline Moncho BSB & 5 & 40 & 20 & 40 & 30 & $\cdots$ & $\cdots$ & $\ldots$ & $\ldots$ \\
\hline Nambu & 20 & 40 & 40 & 40 & so & $\cdots$ & $\cdots$ & $\cdots$ & $\cdots$ \\
\hline Paraguay 281 & 20 & 40 & 20 & 40 & 30 & 20 & 40 & 40 & 40 \\
\hline PAT 24 & 10 & 30 & 20 & 30 & 30 & $\ldots$ & $\ldots$ & $\cdots$ & $\ldots$ \\
\hline PAT 7219 & 10 & 40 & 30 & so & 30 & $\cdots$ & $\cdots$ & $\cdots$ & $\ldots$ \\
\hline BR I & 10 & 40 & 20 & 40 & 30 & $\cdots$ & $\cdots$ & $\cdots$ & $\ldots$ \\
\hline BR 2 & 10 & 40 & 20 & 40 & 30 & $\cdots$ & $\cdots$ & $\cdots$ & $\cdots$ \\
\hline Tucano & 30 & $\ldots$ & $\ldots$ & $\cdots$ & $\cdots$ & $\ldots$ & $\ldots$ & $\cdots$ & $\cdots$ \\
\hline Anahuac & $\ldots$ & 50 & 30 & 40 & 40 & 50 & 20 & 40 & 50 \\
\hline Charrua & $\cdots$ & 40 & 30 & 40 & 30 & $\ldots$ & $\cdots$ & $\cdots$ & $\cdots$ \\
\hline Cocoraque & $\cdots$ & 40 & 40 & 50 & 40 & 30 & 40 & 30 & 50 \\
\hline IAPAR 3 & $\cdots$ & 40 & 30 & 60 & 40 & $\cdots$ & $\cdots$ & $\cdots$ & $\cdots$ \\
\hline Jandaia & $\cdots$ & 40 & 40 & 40 & 50 & $\cdots$ & $\cdots$ & $\cdots$ & $\cdots$ \\
\hline PAT 7392 & $\cdots$ & 40 & 20 & 30 & 30 & $\cdots$ & $\cdots$ & $\cdots$ & $\cdots$ \\
\hline IAC $21\left(^{*}\right)$ & $\cdots$ & $\cdots$ & $\cdots$ & $\cdots$ & $\cdots$ & $\cdots$ & $\cdots$ & $\cdots$ & $\cdots$ \\
\hline IAC $23\left(^{(*)}\right.$ & $\cdots$ & $\cdots$ & $\cdots$ & $\cdots$ & $\cdots$ & $\cdots$ & $\cdots$ & $\cdots$ & $\cdots$ \\
\hline IAC $24\left(^{*}\right)$ & $\cdots$ & $\ldots$ & $\cdots$ & $\cdots$ & $\cdots$ & $\cdots$ & $\cdots$ & $\cdots$ & $\cdots$ \\
\hline
\end{tabular}

(*) Participaram apenas dos ensaios em 1984, quando nāo ocorreu a moléstia.

\section{CONCLUSÕES}

1. Os anos foram altamente influenciados pelas diferenças climáticas ocorridas no quadriênio, sendo 1981 considerado ideal para a cultura quanto a sua condição climatológica no período estudado.

2. Entre os cultivares considerados suscetiveis a toxicidade do aluminio, não houve destaque para a produtividade de grãos, em comparação ao 'BH 1146', testemunha.

3. Entre os cultivares com tolerância ao aluminio tóxico, destacaram-se o PAT 72247, o CEP 7780 e o BH 1146. 
4. Os cultivares Anahuac, Sparrow"S", CEP 74138, CEP 7780, R 30464-77, CNT 8, EI Pato e INIA 66 apresentaram boa resistência à ferrugem do colmo.

5. Para a ferrugem-da-folha, destacou-se como resistente o 'IAPAR 1 Mitacoré; já o 'Alondra 46', que apresentava boa resistência, demonstrou-se altamente suscetivel.

6. Para a helmintosporiose, houve uma variação de ataque, muito dependente das condições climáticas de cada ano; por ocasião de maior ocorrência, não houve destaque para nenhum cultivar.

\title{
SUMMARY
}

\author{
EVALUATION OF WHEAT GENOTYPES IN THE REGION \\ OF PARANAPANEMA VALLEY, STATE OF SÃO PAULO, BRAZIL \\ DURING THE PERIOD 1981-1984
}

\begin{abstract}
Wheat cultivars were evaluated on experiments in soils with and without aluminum toxicity in the Paranapanema Valley, in the State of São Paulo, Brazil, during the period 1981-1984. The characteristics studied were related to reactions to the agent of stem and leaf rusts, reaction to Helminthosporium sp. and the effect of climatic conditions. There was no difference in grain yield among the cultivars presenting sensitivity to aluminum toxicity, however the tolerant cultivars PAT 72247, CEP 7780 and BH 1146 showed good performance in relation to grain yield. The cultivars Anahuac, Sparrow "S", CEP 74138, CEP 7780, R 30464-77, CNT 8, El Pato and INIA 66 presented resistance to stem rust. The cultivar IAPAR 1 (Mitacoré) showed resistance to leaf rust. Alondra 46 classified as resistant to leaf rust until 1982, had the resistance broke down in 1983. The occurrence of leaf diseases caused by Helminthosporium sp. was variable during the period of study increasing its frequency when the plants were submitted to a high humidity period after the pathogen inoculation. The climatic conditions were very different to compare data from different years.
\end{abstract}

Index terms: wheat, genotypes, grain yield, leaf and stem rusts, Helminthosporium sp.

\section{REFERÊNCIAS BIBLIOGRÁFICAS}

BARCELOS, A.L. \& AITA, L. Ferrugem da folha do trigo no Brasil em 1982 e 1983: ocorrência e virulência. In: REUNIĀO NACIONAL DE PESQUISA DE TRIGO, 13., Cruz Alta, 1984. Anais. p.82.

BARROS, B.C.; VALARINI, P.J.; CASTRO, J.L.; FERREIRA FILHO, A.W.P.; LASCA, C.C. \& OLIVEIRA, D.A. Resultados de ensaios de controle químico das doenças de trigo desenvolvidos em São Paulo, 1981. In: REUNIĀO DE COMISSÃO NORTE-BRASILEIRA DE PESQUISA DE TRIGO, 8., Belo Horizonte, 1982. 13p. (Mimeografado) 
CAMARGO, C.E.O. \& OLIVEIRA, O.F. de. Tolerância de cultivares de trigo a diferentes níveis de alumínio em solução nutritiva solo. Bragantia, Campinas, 40:21-31, 1981.

CAMPINAS. Instituto Agronômico. Trigo e cereais de inverno. In: —_. Tabelas de adubação e de calagem. Campinas, 1977. p.189-191. (Boletim, 209)

COELHO, E.T. Distribuição, prevalência e novas raças fisiologicas de Puccinia graminis tritici, no Brasil e outros países do Cone Sul em 1981, 1982 e 1983 (Parcial). In: REUNIÄO NACIONAL DE PESQUISA DE TRIGO, 13., Cruz Alta, 1984. Anais.' p.69.

DUARTE, A.R. Tecnologia na agricultura: considerações sobre a produtividade do trigo brasileiro. São Paulo, USP, 1974. 144p. Tese (Doutoramento)

EMBRAPA. Centro Nacional de Pesquisa de Trigo. Programa Nacional de Pesquisa. Passo Fundo, 1984. 36p. (Mimeografado)

FELÍCIO, J.C.; BARROS, B.C.; CAMARGO, C.E.O. \& BAR, W.R. Maracaí (IAC 17) e Xavantes (IAC 18): cultivares de trigo para o Estado de São Paulo. Bragantia, Campinas, 42:15-25, 1983.

GOMES, F.P. Curso de estatística experimental. Piracicaba, Escola Superior de Agricultura "Luiz de Queiroz", 1970. 384p.

KALCKMANN, R.E.; ARRUDA, A.A.G.; HOETGEBAUM, F.; POPA, W.; BALDANZI, G. \& GODOY, L.C. de. Regiōes de trigo no Brasil: 1ª aproximação. Rio de Janeiro, Serviço de Informação Agrícola, 1965. 104p. (Estudos Técnicos, 28)

LUZ, W.C. da. Influência do período de umidificação pós-inoculação na reação de cultivares de trigo à mancha foliar (Cochliobolus sativus). In: REUNIÃO NACIONAL DE PESQUISA DE TRIGO, 12., Cascavel, 1982. Anais. p.186-191.

MOTA, F.S. da. Clima e zoneamento para a triticultura no Brasil. In: TRIGO no Brasil. Campinas, Fundação Cargill, 1982. v.1, p.29-92.

MUZZILLI, O.; SANTOS, D.; PALHANO, J.B.; MANETTI FILHO, J.L.A.F.; GARCIA, A. \& CAETANO, A. Tolerância de cultivares de soja e trigo à acidez do solo. Revista Brasileira de Ciência do Solo, Campinas, 2(1):34-40, 1978.

OLIVEIRA, M.A.R. de \& GOMES, L.S. Avaliação da perda em rendimento causada por helmintosporiose em trigo. In: REUNIÄO NACIONAL DE PESQUISA DE TRIGO, 13., Cruz Alta, 1984. Anais. p.65.

POEHLMAN, J.M. Mejoramiento genético del trigo. In: MEJORAMIENTO genético de las cosechas. México, Limusa, 1974. Item 6, p.123-150.

SCHRAM, W.; FULCO, W.S.; SOARES, M.H.G. \& ALMEIDA, A.M.P. Resistência de cultivares de trigo em experimentação ou cultivo no Rio Grande do Sul, às principais doenças fúngicas. Agronomia Sul Rio-Grandense, Porto Alegre, 10:31-39, 1974.

THORNTHWAITE, C.W. \& MATHER, J.R. The water balance. Centerton, N.J., Drexel Institute of Tecnology, 1955. 104p. (Publications in climatology, v.8, n- 1) 